\title{
Hepatocyte-secreted DPP4 in obesity promotes adipose inflammation and insulin resistance
}

Devram S. Ghorpade ${ }^{1}$, Lale Ozcan ${ }^{1}$, Ze Zheng ${ }^{1}$, Sarah M. Nicoloro², Yuefei Shen ${ }^{2}$, Emily Chen ${ }^{3,4}$, Matthias Blüher ${ }^{5}$, Michael P. Czech ${ }^{2} \&$ Ira Tabas $^{1,6}$

\begin{abstract}
Obesity-induced metabolic disease involves functional integration among several organs via circulating factors, but little is known about crosstalk between liver and visceral adipose tissue (VAT) ${ }^{1}$. In obesity, VAT becomes populated with inflammatory adipose tissue macrophages (ATMs) ${ }^{2,3}$. In obese humans, there is a close correlation between adipose tissue inflammation and insulin resistance ${ }^{4,5}$, and in obese mice, blocking systemic or ATM inflammation improves insulin sensitivity ${ }^{6-8}$. However, processes that promote pathological adipose tissue inflammation in obesity are incompletely understood. Here we show that obesity in mice stimulates hepatocytes to synthesize and secrete dipeptidyl peptidase 4 (DPP4), which acts with plasma factor Xa to inflame ATMs. Silencing expression of DPP4 in hepatocytes suppresses inflammation of VAT and insulin resistance; however, a similar effect is not seen with the orally administered DPP4 inhibitor sitagliptin. Inflammation and insulin resistance are also suppressed by silencing expression of caveolin-1 or PAR2 in ATMs; these proteins mediate the actions of DPP4 and factor Xa, respectively. Thus, hepatocyte DPP4 promotes VAT inflammation and insulin resistance in obesity, and targeting this pathway may have metabolic benefits that are distinct from those observed with oral DPP4 inhibitors.
\end{abstract}

In obese mice and humans, a pathway in hepatocytes involving $\mathrm{Ca}^{2+}$-calmodulin-dependent protein kinase II (CaMKII) contributes to both excessive hepatic glucose production and impaired hepatic insulin signalling ${ }^{9-11}$. Inhibition of this pathway by hepatocyte-specific deletion of CaMKII $\gamma$ in diet-induced obese (DIO) mice (Camk $2 g^{f l / f l}$ mice infected with AAV8-TBG-cre, hereafter referred to as H-CaMKII(KO) mice, improves glucose and insulin tolerance ${ }^{9,10}$. Notably, the VAT of these mice had fewer ATMs in crown-like structures (CLS) and decreased Adgre1 mRNA (which encodes the F4/80 glycoprotein) in comparison to VAT of control Camk $2 g^{f l / f l}$ DIO mice (Extended Data Fig. 1a). There was also decreased infiltration of inflammatory Ly6 $\mathrm{C}^{\text {hi }}$ monocytes and lower inflammatory cytokine expression in the VAT of $\mathrm{H}$-CaMKII $(\mathrm{KO})$ mice, with no significant change in blood monocyte number, plasma IL6 or TNF $\alpha$, or liver inflammation (Extended Data Fig. 1b-e). ATF4 expression is regulated downstream of CaMKII signalling and is decreased in the hepatocytes of $\mathrm{H}-\mathrm{CaMKII}(\mathrm{KO}) \mathrm{DIO}$ mice $^{10}$. Restoring ATF4 expression in the livers of mice deficient in hepatocyte CaMKII using an adenovirus vector restored VAT inflammation without affecting liver inflammation (Extended Data Fig. 1f, g). Conversely, hepatocyte-specific deletion of ATF4 in DIO mice lowered VAT inflammation (Extended Data Fig. 1h). Therefore, we hypothesized that, in obesity, activation of CaMKII and ATF4 in hepatocytes induces the secretion of a circulatory factor (a 'hepatokine') that promotes VAT inflammation.

To test this hypothesis, we developed an ex vivo assay in which cells from the stromal vascular fraction (SVF) of VAT are incubated with plasma from the above mouse models, and Mcp1 (also known as Ccl2) and Il6 mRNA are quantified as markers of inflammation. Because VAT macrophages are a likely target of the putative hepatokine, we predicted that cells from the SVF of VAT from obese mice, which harbour more of these macrophages ${ }^{4,5}$ (Extended Data Fig. 2a), would show an increased response in this assay in comparison to SVF cells from lean mice. Plasma from obese mice would also be expected to evoke a more potent response in the assay than plasma from lean mice. Consistent with this hypothesis, plasma from DIO mice induced higher expression of both Mcp1 and Il6 mRNA than plasma from lean mice in SVF cells from DIO mice, but did not have this effect on SVF cells from lean mice. Furthermore, the inflammatory activity of plasma from DIO mice was due to the macrophage component of SVF cells (Fig. 1a and Extended Data Fig. 2b). DIO mouse plasma was also able to induce $M c p 1$ expression in peritoneal and bone marrow-derived macrophages (BMDMs; Extended Data Fig. 2c). Most importantly, plasma from H-CaMKII-(KO) DIO mice was less able to induce Mcp1 expression in SVF than plasma from either wild-type DIO mice or $\mathrm{H}$-CaMKII(KO) mice in which hepatic ATF4 expression was restored (Fig. 1b). These data suggest that, in obesity, activation of hepatocyte CaMKII and ATF4 induce one or more secretory factors that promote VAT-macrophage inflammation.

The Mcp1-inducing factor in DIO mouse plasma was heat labile (Extended Data Fig. 2d), suggesting that it may be a protein. We performed size-exclusion gel-filtration fast protein liquid chromatography (FPLC) with DIO mouse plasma (Extended Data Fig. 2e) and tested eluted fractions for their ability to induce Mcp1 expression in SVF cells. There was a peak of activity in three fractions in the $125-200 \mathrm{kDa}$ range (Extended Data Fig. 2f). The active fraction F44 and inactive fractions F42 and F46 were analysed by liquid chromatography-tandem mass spectrometry (LC-MS/MS) to identify peptides that were more abundant in F44 than in F42 and F46 (Supplementary Table 2a). Peptides corresponding to DPP4 (CD26) met these criteria (Extended Data Fig. $2 \mathrm{~g}$ ). DPP4 is a dipeptidyl protease that can exist as either a cell membrane protein or a soluble plasma protein ${ }^{12}$. DPP 4 concentration correlates with body mass index and insulin resistance in humans ${ }^{13,14}$, and we found that DPP4 activity was higher in the plasma of DIO mice than in that of lean mice (Extended Data Fig. 2h).

Consistent with a role for DPP4, the Mcp1- and Il6-inducing activity of DIO mouse plasma could be suppressed by an inhibitor of DPP4 (Fig. 1c and Extended Data Fig. 2i). Furthermore, DPP4 activity in plasma and Dpp4 mRNA expression in liver, but not in VAT, correlated exactly with VAT inflammation in vivo and plasma SVF-inflammatory activity ex vivo in wild-type and $\mathrm{H}$-CaMKII $(\mathrm{KO})$ mice as well as in $\mathrm{H}$-CaMKII(KO) mice in which hepatic ATF4 expression was restored (Fig. 1d and Extended Data Fig. 2j). Moreover, restoration of hepatic DPP4 expression in $\mathrm{H}-\mathrm{CaMKII}(\mathrm{KO})$ mice restored DPP4 activity in plasma and VAT inflammation without changing body weight (Extended Data Fig. 3a, b). Consistent with the role of ATF4, we 

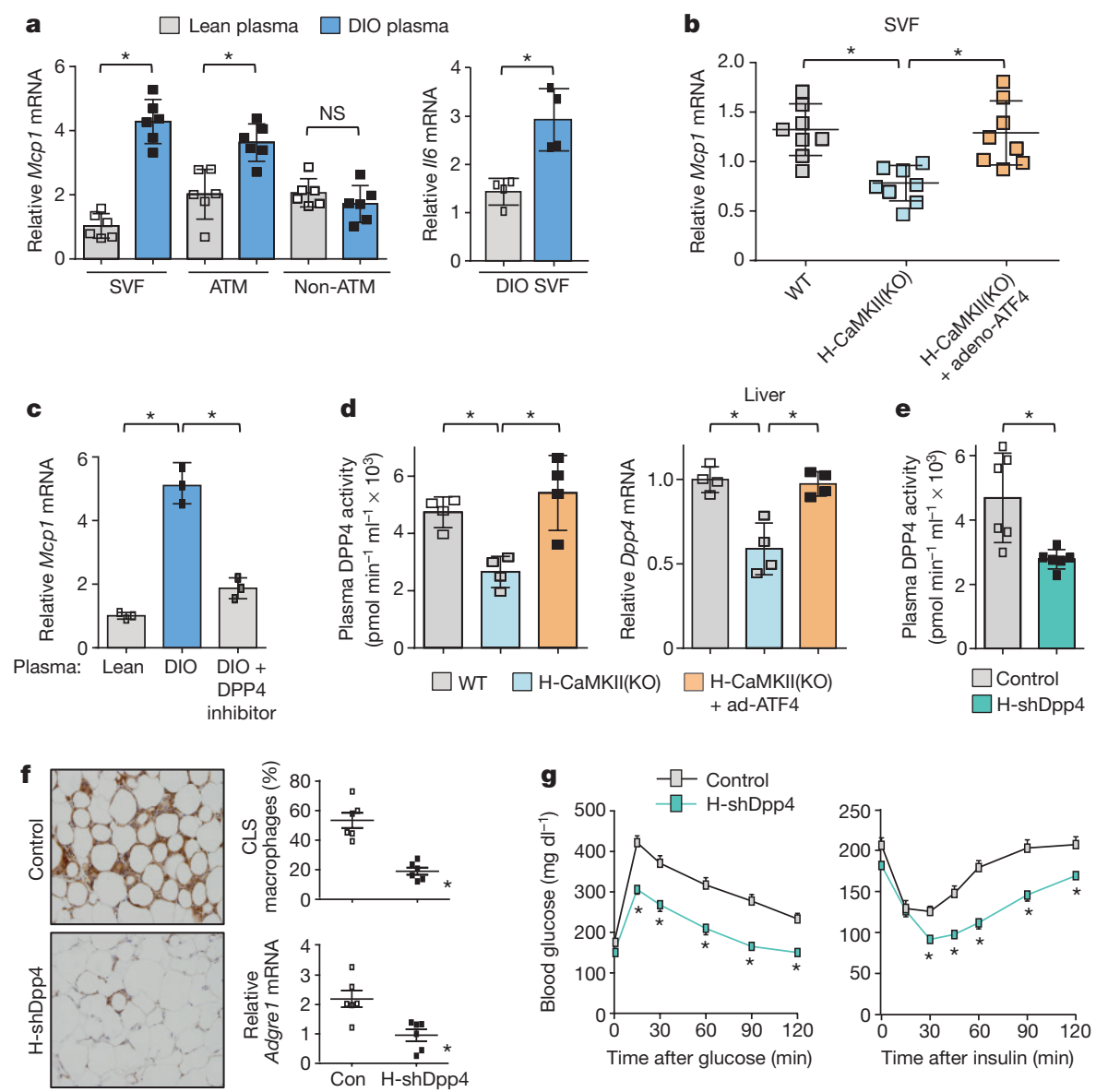

Figure 1 | Hepatocyte-specific DPP4 silencing suppresses VAT inflammation and improves insulin sensitivity in DIO mice. a, VAT SVF cells from DIO mice, and ATMs and non-ATMs isolated from SVF, were incubated for $4 \mathrm{~h}$ with medium containing $10 \%$ (vol/vol) plasma from lean or DIO mice and then assayed for Mcp1 or Il6 mRNA ( $n=4-6$ mice per group; ${ }^{*} P<0.05$ by two-tailed Student's $t$-test). $\mathbf{b}$, VAT SVF cells from DIO mice were incubated with plasma from the three DIO models described in Extended Data Fig. 1f, g and then assayed for Mcp1 mRNA. WT, wild type. $n=8$ mice per group; mean \pm s.e.m.; $* P<0.05$ by one-way analysis of variance (ANOVA). c, SVF cells from DIO mice were incubated with lean or DIO mouse plasma that was pre-treated for $1 \mathrm{~h}$ with or without $10 \mu$ M DPP4 inhibitor KR62436 and then assayed for Mcp1 mRNA $(n=3$

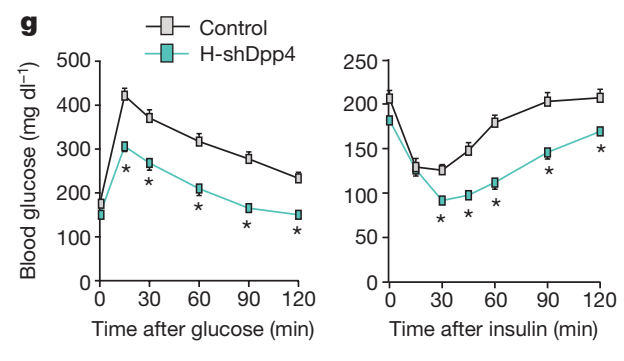

technical replicates; mean \pm s.e.m.; $* P<0.05$ by one-way ANOVA). d, The three groups of mice from Extended Data Fig. 1f, g were assayed for plasma DPP4 activity and hepatic Dpp 4 mRNA ( $n=4$ mice per group; mean \pm s.e.m.; $* P<0.05$ by one-way ANOVA). e-g, Sixteen-week-old mice previously fed the DIO diet for 10 weeks were injected intravenously with AAV8-H1-shDpp4 (H-shDpp4) or control AAV8-H1 vector (control; or con, in f). The mice were analysed after four weeks as follows. e, Plasma DPP4 activity. f, CLS macrophages and Adgre 1 mRNA in VAT, with representative images of F4/80-stained VAT. g, Blood glucose after intraperitoneal glucose or insulin ( $n=6$ mice per group; mean \pm s.e.m.; $* P<0.05$ by two-tailed Student's $t$-test).

found an ATF4-consensus site in exon 1 of the Dpp4 gene. Chromatin immunoprecipitation (ChIP) analysis revealed lower levels of ATF4 occupancy at this site in the livers of H-CaMKII(KO) DIO mice versus those of wild-type DIO or $\mathrm{H}$-CaMKII(KO) DIO mice in which hepatocyte ATF4 expression was restored (Extended Data Fig. 3c).

We infected DIO mice with an adeno-associated virus 8 (AAV8) that encoded a short hairpin RNA (shRNA) construct targeting mouse Dpp4 driven by the $\mathrm{H} 1$ promoter. AAV8-H1-shDpp4 specifically silences DPP4 in hepatocytes but not in VAT or other tissues (Extended Data Fig. 3d, e); we refer to the vector as $\mathrm{H}$-shDpp4 to reflect its hepatocyte specificity. H-shDpp4-treated DIO mice had significantly decreased plasma DPP4 activity (Fig. 1e), indicating that hepatocytes are a source of circulating DPP4 in obesity. H-shDpp4-treated DIO mice also had decreased numbers of CLS macrophages in the VAT and decreased levels of inflammatory cytokine mRNAs in VAT and VAT ATMs (Fig. $1 \mathrm{f}$ and Extended Data Fig. 4a, b). However, these mice did not differ from control vector-treated DIO mice with regard to inflammation in inguinal or brown fat, plasma cytokines, body weight, food intake or liver or adipose tissue weight (Extended Data Fig. 4c-g). H-shDpp4treated DIO mice also exhibited improved glucose homeostasis and increased insulin-induced AKT phosphorylation (p-AKT) in VAT and

liver (Fig. 1g and Extended Data Fig. 4h, i). Unlike DPP4 inhibition ${ }^{15}$, silencing of hepatocyte DPP4 did not increase levels of active plasma incretins (Extended Data Fig. 4j). Similar data were obtained when $o b / o b$ mice were treated with the H-shDpp4 (Extended Data Fig. 5a-h). By contrast, treatment of lean mice with the H-shDpp4 had no effect on metabolism (Extended Data Fig. 5i-m), and DPP4 silencing did not improve insulin signalling in palmitate-treated primary hepatocytes (Extended Data Fig. 6a). Moreover, consistent with the idea that adipocyte-derived non-esterified fatty acids (NEFA) may contribute to inflammation-induced insulin resistance ${ }^{16}, \mathrm{H}$-shDpp4 treatment lowered plasma NEFA in obese but not in lean mice (Extended Data Fig. 6b). These combined data suggest that the improvement in metabolism in DIO mice treated with H-shDpp4 is caused by suppression of VAT inflammation, which improves insulin signalling in hepatocytes and VAT as a secondary effect rather than via a direct effect on insulin signalling in hepatocytes.

Oral DPP4 inhibitors do not lower plasma insulin in insulin-resistant, hyperinsulinaemic subjects ${ }^{17-22}$. A recent study suggested that in obese mice, oral DPP4 inhibitors function by inhibiting gut endothelial DPP4 ${ }^{23}$. We tested the hypothesis that oral DPP4 inhibition and hepatocyte DPP4 silencing have fundamentally different effects on adipose 


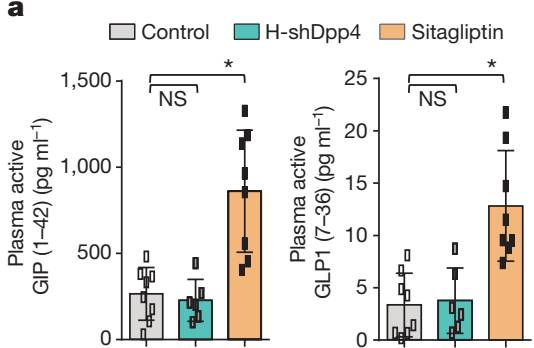

b
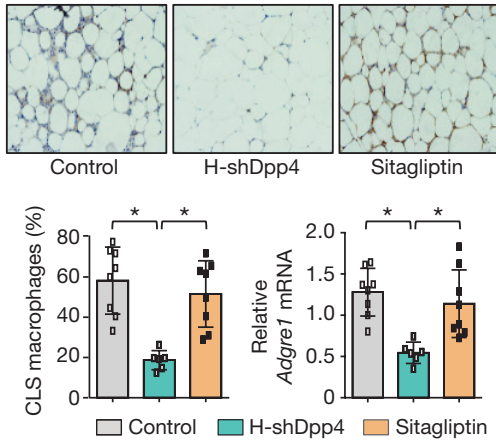

C

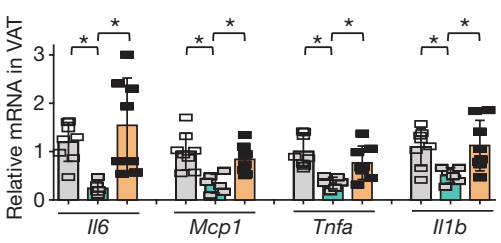

d

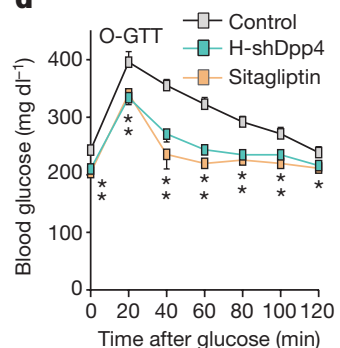

a

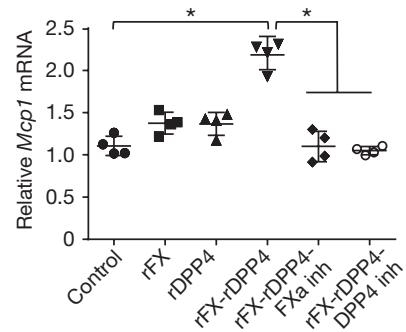

c d

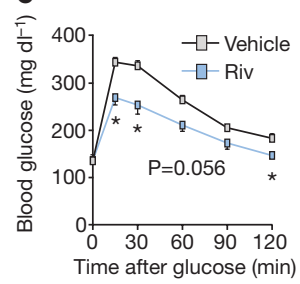

d
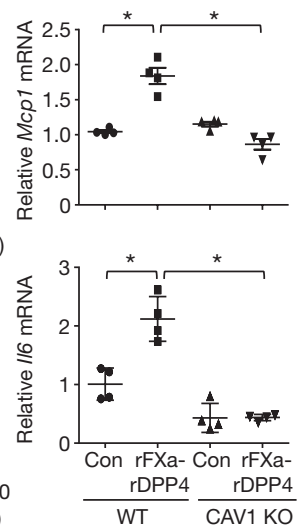

b
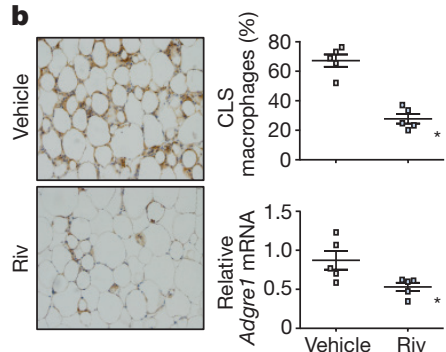
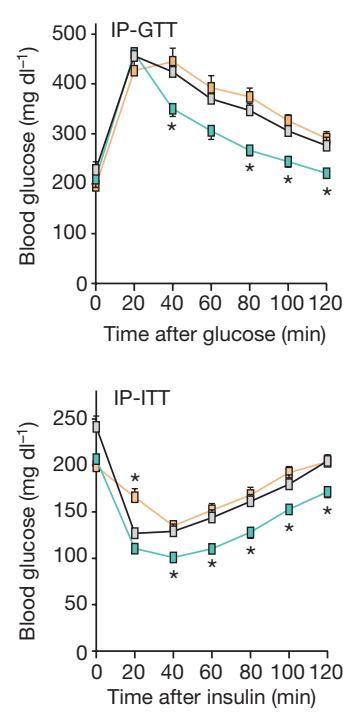

e

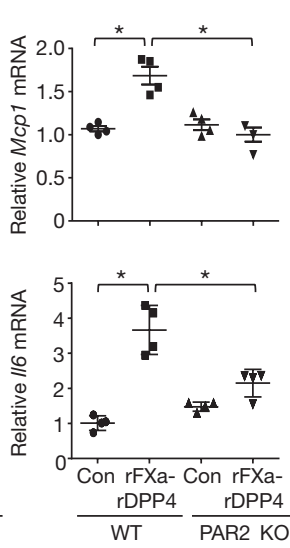

Figure 3 | DPP4 and FXa synergistically activate inflammatory signalling in macrophages. a, BMDMs were pretreated with or without $10 \mu \mathrm{M}$ FXa inhibitor rivaroxaban or $10 \mu \mathrm{M}$ DPP4 inhibitor KR62436, followed by incubation for $4 \mathrm{~h}$ with $\mathrm{rFX}$ or $\mathrm{rDPP} 4$ alone or in combination. Mcp1 mRNA was then quantified. $n=4$ technical replicates per group; mean \pm s.e.m.; $* P<0.05$ by one-way ANOVA. b, c, Sixteen-week-old mice previously fed the DIO diet for 10 weeks were treated for 20 days with $2 \mathrm{mg} \mathrm{kg}^{-1}$ oral rivaroxaban twice daily (Riv) or vehicle control and analysed as follows. b, CLS macrophages and Adgre1 mRNA in VAT, with representative images of F4/80-stained VAT. c, Blood glucose after intraperitoneal glucose or insulin $(n=5$ mice per group; mean \pm s.e.m.; $* P<0.05$ by two-tailed Student's $t$-test). d, e, BMDMs from wild-type, CAV1 knockout (CAV1 KO) or PAR2 knockout (PAR2 KO) mice were incubated for $4 \mathrm{~h}$ with or without $\mathrm{rFX}$ and $\mathrm{rDPP} 4$ and then assayed for $M c p 1$ and $I l 6$ mRNA. $n=4$ technical replicates per group; mean \pm s.e.m.; $* P<0.05$ by two-way ANOVA.

inflammation and glucose metabolism: we directly compared treatment with the oral DPP4 inhibitor sitagliptin with silencing of hepatocyte DPP4 in DIO mice. After four weeks, neither treatment affected body weight; both treatments lowered plasma DPP4 activity to a similar degree and neither treatment affected DPP4 activity in VAT (Extended Data Fig. 6c, d). However, H-shDpp4, but not sitagliptin, decreased hepatic DPP4 protein and hepatocyte DPP4 activity (Extended Data Fig. 6e). Conversely, sitagliptin, but not $\mathrm{H}$-shDpp4, increased plasma incretins (Fig. 2a), consistent with our shDpp4 data and the known action of oral DPP4 inhibitors. Notably, H-shDpp4, but not sitagliptin, suppressed VAT inflammation (Fig. 2b, c), and although both treatments lowered blood glucose and improved oral glucose tolerance, only H-shDpp4 lowered plasma insulin, improved glucose response to intraperitoneal glucose and insulin, showed evidence of increased insulin-induced p-AKT in VAT and liver and lowered plasma NEFA (Fig. 2d and Extended Data Fig. 6f-h). Consistent with these results, both sitagliptin and H-shDpp4 treatments lowered plasma DPP4 activity and blood glucose, but only $\mathrm{H}$-shDpp4 decreased plasma insulin levels after 11 weeks, without a change in body weight (Extended Data Fig. 6i). Thus, DPP4 inhibition by orally administered sitagliptin and hepatocyte DPP4 silencing have fundamentally different effects on VAT inflammation and metabolism.

Next, we investigated the mechanism of DPP4-induced VAT macrophage inflammation. Although recombinant DPP4 (rDPP4) alone was unable to induce Mcp1 or Il6 expression in SVF, it did so when

added to either DIO mouse plasma that had been immunodepleted of DPP4 or plasma from DIO mice that had been treated with H-shDpp4 (Extended Data Fig. 7a, b). To determine whether an additional plasma factor was needed for DPP4 to promote SVF inflammation, plasma from DIO mice was immunodepleted of DPP4 and then fractionated using FPLC. Two fractions (F43 and F44) were able to induce Mcp1 in SVF cells in the presence of rDPP4, but not in its absence (Extended Data Fig. 7c). Fraction F44 and the inactive fractions F42 and F46 were then analysed by LC-MS/MS to identify peptides that were more abundant in F44 versus F42 and F46 (Supplementary Table 2b). Peptides corresponding to plasma factor X met these criteria (Extended Data Fig. $8 \mathrm{a})$. Factor X can trigger inflammation in endothelial cells and leukocytes $^{24,25}$, and we found that the ability of DIO plasma to induce Mcp1 expression in SVF cells or macrophages was abrogated by rivaroxaban, an inhibitor of factor Xa (FXa) (Extended Data Fig. 8b). Furthermore, whereas recombinant factor X ( $\mathrm{rFX}$ ) or $\mathrm{rDPP} 4$ alone led to only very small increases in Mcp1 and Il6 expression in macrophages, combined treatment with both $\mathrm{rFX}$ and $\mathrm{rDPP} 4$ was much more potent, and this inflammatory activity was inhibited by either rivaroxaban or a DPP4 inhibitor (Fig. 3a and Extended Data Fig. 8c). These data also imply that factor X is converted to FXa by macrophages ${ }^{26}$. Using recombinant $\mathrm{FXa}(\mathrm{rFXa})$ directly, we showed that $\mathrm{rFXa}$ and $\mathrm{rDPP} 4$ together were able to induce Mcp1 and Il6 expression in DIO mouse SVF cells but not in 
lean mouse SVF cells (Extended Data Fig. 8d). Treatment of DIO mice with rivaroxaban lowered VAT inflammation and plasma NEFA, and improved metabolism, without affecting body weight (Fig. 3b, c and Extended Data Fig. 8e-j).

To understand how the combination of DPP4 and FXa induces $M c p 1$ expression, we pre-treated macrophages with rFXa and then, after removing the $\mathrm{rFXa}$ and inhibiting residual activity with rivaroxaban, treated the cells with rDPP4. We also conducted the reverse experiment by pre-treating the cells with rDPP4 and then, after removing the rDPP4 and inhibiting residual activity with a DPP4 inhibitor, treated the cells with rFXa. Both protocols increased Mcp1 and Il6 mRNA expression (Extended Data Fig. 9a, b), suggesting that neither a complex between FXa and DPP4 nor a specifically ordered priming event is needed. We therefore hypothesized that each factor alone partially activates inflammatory signalling to a sub-threshold level, and that the threshold is reached only when both factors are present. Inflammation induced by rDPP4 and rFXa was markedly reduced in macrophages from mice lacking either caveolin-1 (CAV1), which mediates the inflammatory effects of DPP4 on antigen-presenting cells ${ }^{27}$, or PAR2, a cell-surface receptor that mediates the inflammatory effects of FXa in macrophages ${ }^{28}$ (Fig. 3d, e). Similar results were observed in VAT-derived ATMs from DIO mice, and human macrophages treated with inhibitors of CAV1 or PAR2 (Extended Data Fig. 9c, d). Consistent with the signalling-threshold hypothesis, rDPP4 alone increased phosphorylation of two reported CAV1 signalling intermediates, IRAK1 and TAK1 (Extended Data Fig. 9e), and rFXa alone increased phosphorylation of the PAR2 signalling intermediate RAF1 (Extended Data Fig. 9f), but only combined treatment with rDPP4 and rFXa was able to activate two distal inflammatory signalling molecules, ERK1/2 and NF- $\kappa$ B (Extended Data Fig. 9g). Pharmacological inhibition of these signalling molecules abolished rDPP4-rFXa-induced Mcp 1 and Il6 expression (Extended Data Fig. $9 c, h-j)$. Together, these data suggest that DPP4 and FXa activate two separate upstream pathways that synergistically stimulate ERK1/2 and NF- $\kappa$ B to induce Mcp 1 and Il6 in ATMs.

Next, we determined whether inhibition of the above pathways in ATMs could ameliorate VAT inflammation and improve metabolism in obese mice. Genes can be silenced specifically in ATMs in $o b / o b$ mice by intraperitoneal administration of short interfering RNA ( siRNA) encapsulated in micrometer-sized glucan shells, referred to as glucan-encapsulated siRNA particles (GERPs) ${ }^{8}$. Using this method, we determined the effect of decreasing Par2 and Cav1 expression in ATMs on VAT inflammation and glucose metabolism in $o b / o b$ mice. In a pilot experiment using FITC-labelled glucan shells in $o b / o b$ mice, we verified their increased localization to macrophages in VAT (ATMs) relative to macrophages in other organs (Extended Data Fig. 10a). We then treated $o b / o b$ mice with GERPs containing scrambled RNA (control), or siRNA targeting PAR2 or CAV1, over a 12-day period. Body weight was similar in all three cohorts (Extended Data Fig. 10b). PAR2 and CAV1 levels were decreased by the respective GERPs in ATMs but not in spleen (Extended Data Fig. 10c). Notably, both GERPs lowered VAT inflammation, improved glucose intolerance, increased insulin-induced $\mathrm{p}$-AKT in VAT and liver and decreased plasma NEFA (Fig. 4a-d and Extended Data Fig. 10d, e).

These findings emphasize the important role of crosstalk between hepatocytes and adipose tissue in metabolic disease (Fig. 4e) and the distinct effects of silencing hepatocyte DPP4 expression versus DPP4 inhibition by oral inhibitors, which are widely used to treat type 2 diabetes. Although sitagliptin has been reported to lower adipose inflammation in obese mice ${ }^{29}$, the drug was administered to young mice when they started receiving a high-fat diet, leading to an approximately $25 \%$ decrease in body weight in comparison to untreated mice. In our study, the drug was given to adult mice after obesity and VAT inflammation had developed, and it had no effect on body weight. We speculate that inhibition of circulating DPP4 activity does not block VAT inflammation in adult obese mice because some other action of oral DPP4
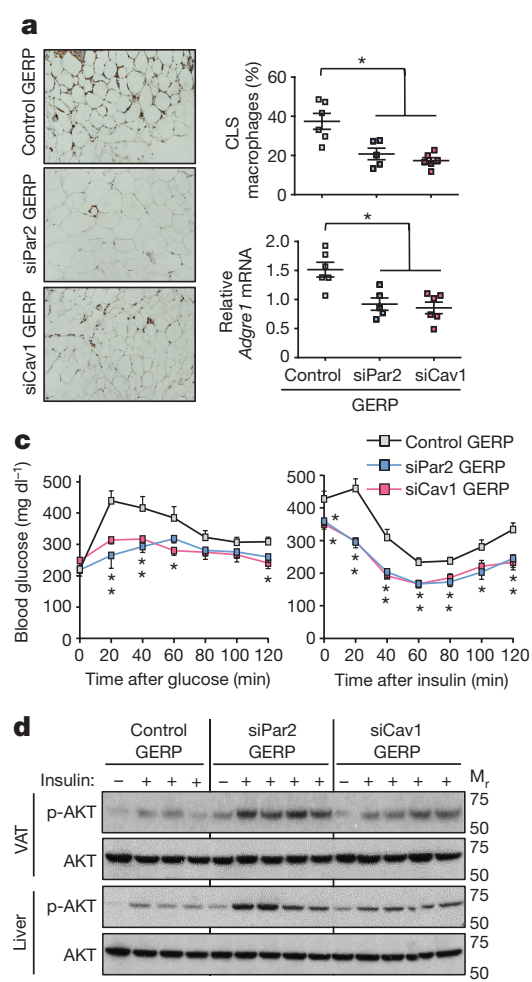

Figure 4 | Silencing PAR2 or CAV1 in ATMs in obese mice lowers VAT inflammation and improves response to insulin. a-d, Five-week-old, chow-fed $o b / o b$ mice were injected intraperitoneally once a day for 12 days with $0.2 \mathrm{mg}$ GERPs containing scrambled siRNA (control), Par2 siRNA (siPar2) or Cav1 siRNA (siCav1). Twenty-four hours after the last injection, the mice were analysed as follows. a, CLS macrophages and Adgre 1 mRNA in VAT, with representative images of F4/80-stained VAT. b, Blood glucose and plasma insulin $5 \mathrm{~h}$ after food withdrawal. c, Blood glucose after intraperitoneal glucose or insulin. d, Total AKT and p-AKT in VAT and liver after portal vein insulin injection. $n=5-6$ mice per group; $* P<0.05$ by two-tailed Student's $t$-test for each siRNA GERP versus control GERP. For gel source data, see Supplementary Fig. 1. e, Summary scheme: obesity triggers a $\mathrm{Ca}^{2+}-\mathrm{CaMKII}-\mathrm{ATF} 4$ pathway in hepatocytes, leading to induction of DPP4 and secretion of soluble DPP4 (sol-DPP4). Soluble DPP4 activates a CAV1-IRAK1-TAK1 pathway in ATMs, which, in combination with PAR2-RAF1 activation by FXa in ATMs, promotes ERK1/2-NF-kB-mediated inflammation. VAT inflammation exacerbates glucose intolerance, impaired insulin signalling in adipose and liver and hyperinsulinaemia.

inhibition in that setting, perhaps related to the increase in plasma insulin ${ }^{30}$, counteracts the anti-inflammatory effect of lowering plasma DPP4 activity. While future work is needed to understand the role of the hepatocyte DPP4-FXa pathway in obese, insulin-resistant humans, we have preliminary data that shows that a high percentage of obese subjects with fat inflammation and a high homeostasis model assessment (HOMA) index have inflammatory activity in their plasma that is dependent on DPP4, FXa and PAR2, whereas plasma from obese subjects without fat inflammation and insulin resistance do not show this dependency. In conclusion, the identification of this pathway provides insight into how organ crosstalk can exacerbate metabolic disarray in obesity and raises the possibility that therapeutic silencing of hepatocyte DPP4 - via hepatocyte-targeted siRNA ${ }^{31}$, for example-may have benefits for obesity-induced insulin resistance that are not achievable through currently available oral DPP4 inhibitors.

Online Content Methods, along with any additional Extended Data display items and Source Data, are available in the online version of the paper; references unique to these sections appear only in the online paper.

Received 27 June 2017; accepted 14 February 2018.

Published online 21 March 2018. 
1. Dasgupta, S. et al. NF-kB mediates lipid-induced fetuin-A expression in hepatocytes that impairs adipocyte function effecting insulin resistance. Biochem. J. 429, 451-462 (2010).

2. Weisberg, S. P. et al. Obesity is associated with macrophage accumulation in adipose tissue. J. Clin. Invest. 112, 1796-1808 (2003).

3. $\mathrm{Xu}, \mathrm{H}$. et al. Chronic inflammation in fat plays a crucial role in the development of obesity-related insulin resistance. J. Clin. Invest. 112, 1821-1830 (2003)

4. Hardy, O. T. et al. Body mass index-independent inflammation in omental adipose tissue associated with insulin resistance in morbid obesity. Surg. Obes. Relat. Dis. 7, 60-67 (2011).

5. Blüher, M. Adipose tissue inflammation: a cause or consequence of obesity-related insulin resistance? Clin. Sci. (Lond.) 130, 1603-1614 (2016).

6. Hotamisligil, G. S., Shargill, N. S. \& Spiegelman, B. M. Adipose expression of tumor necrosis factor-a: direct role in obesity-linked insulin resistance. Science 259, 87-91 (1993)

7. Lumeng, C. N. \& Saltiel, A. R. Inflammatory links between obesity and metabolic disease. J. Clin. Invest. 121, 2111-2117 (2011).

8. Aouadi, M. et al. Gene silencing in adipose tissue macrophages regulates whole-body metabolism in obese mice. Proc. Natl Acad. Sci. USA 110, 8278-8283 (2013)

9. Ozcan, L. et al. Calcium signaling through CaMKII regulates hepatic glucose production in fasting and obesity. Cell Metab. 15, 739-751 (2012).

10. Ozcan, L. et al. Activation of calcium/calmodulin-dependent protein kinase II in obesity mediates suppression of hepatic insulin signaling. Cell Metab. 18, 803-815 (2013).

11. Ozcan, L. et al. Hepatocyte DACH1 is increased in obesity via nuclear exclusion of HDAC4 and promotes hepatic insulin resistance. Cell Reports $\mathbf{1 5}$ 2214-2225 (2016)

12. Boonacker, E. \& Van Noorden, C. J. The multifunctional or moonlighting protein CD26/DPPIV. Eur. J. Cell Biol. 82, 53-73 (2003).

13. Kirino, Y., Sei, M., Kawazoe, K., Minakuchi, K. \& Sato, Y. Plasma dipeptidyl peptidase 4 activity correlates with body mass index and the plasma adiponectin concentration in healthy young people. Endocr. J. 59, 949-953 (2012).

14. Lamers, D. et al. Dipeptidyl peptidase 4 is a novel adipokine potentially linking obesity to the metabolic syndrome. Diabetes 60, 1917-1925 (2011)

15. Drucker, D. J. \& Nauck, M. A. The incretin system: glucagon-like peptide-1 receptor agonists and dipeptidyl peptidase-4 inhibitors in type 2 diabetes. Lancet 368, 1696-1705 (2006).

16. Guilherme, A., Virbasius, J. V., Puri, V. \& Czech, M. P. Adipocyte dysfunctions linking obesity to insulin resistance and type 2 diabetes. Nat. Rev. Mol. Cell Biol. 9, 367-377 (2008)

17. Ristic, S., Byiers, S., Foley, J. \& Holmes, D. Improved glycaemic control with dipeptidyl peptidase-4 inhibition in patients with type 2 diabetes: vildagliptin (LAF237) dose response. Diabetes Obes. Metab. 7, 692-698 (2005).

18. Aschner, P. et al. Effect of the dipeptidyl peptidase-4 inhibitor sitagliptin as monotherapy on glycemic control in patients with type 2 diabetes. Diabetes Care 29, 2632-2637 (2006).

19. Raz, l. et al. Efficacy and safety of the dipeptidyl peptidase-4 inhibitor sitagliptin as monotherapy in patients with type 2 diabetes mellitus. Diabetologia 49, 2564-2571 (2006).

20. Kutoh, E. \& Ukai, Y. Alogliptin as an initial therapy in patients with newly diagnosed, drug naïve type 2 diabetes: a randomized, control trial. Endocrine 41, 435-441 (2012).

21. Kadowaki, T. \& Kondo, K. Efficacy, safety and dose-response relationship of teneligliptin, a dipeptidyl peptidase-4 inhibitor, in Japanese patients with type 2 diabetes mellitus. Diabetes Obes. Metab. 15, 810-818 (2013).
22. Jung, C. H. et al. A randomized, double-blind, placebo-controlled, phase I clinical trial to investigate the efficacy and safety of oral DA-1229 in patients with type 2 diabetes mellitus who have inadequate glycaemic control with diet and exercise. Diabetes Metab. Res. Rev. 31, 295-306 (2015).

23. Mulvihill, E. E. et al. Cellular sites and mechanisms linking reduction of dipeptidyl peptidase-4 activity to control of incretin hormone action and glucose homeostasis. Cell Metab. 25, 152-165 (2017).

24. Senden, N. H. et al. Factor $\mathrm{Xa}$ induces cytokine production and expression of adhesion molecules by human umbilical vein endothelial cells. J. Immunol. 161, 4318-4324 (1998).

25. Busch, G. et al. Coagulation factor Xa stimulates interleukin-8 release in endothelial cells and mononuclear leukocytes: implications in acute myocardial infarction. Arterioscler. Thromb. Vasc. Biol. 25, 461-466 (2005).

26. McGee, M. P., Wallin, R., Wheeler, F. B. \& Rothberger, H. Initiation of the extrinsic pathway of coagulation by human and rabbit alveolar macrophages: a kinetic study. Blood 74, 1583-1590 (1989).

27. Ohnuma, K. et al. CD26 mediates dissociation of Tollip and IRAK-1 from caveolin-1 and induces upregulation of CD86 on antigen-presenting cells. Mol. Cell. Biol. 25, 7743-7757 (2005).

28. Zuo, P. et al. Factor Xa induces pro-inflammatory cytokine expression in RAW 264.7 macrophages via protease-activated receptor-2 activation. Am. J. Transl. Res. 7, 2326-2334 (2015).

29. Dobrian, A. D. et al. Dipeptidyl peptidase IV inhibitor sitagliptin reduces local inflammation in adipose tissue and in pancreatic islets of obese mice. Am. J. Physiol. Endocrinol. Metab. 300, E410-E421 (2011).

30. Pedersen, D. J. et al. A major role of insulin in promoting obesity-associated adipose tissue inflammation. Mol. Metab. 4, 507-518 (2015).

31. Fitzgerald, K. et al. A highly durable RNAi therapeutic inhibitor of PCSK9. N. Engl. J. Med. 376, 41-51 (2017)

Supplementary Information is available in the online version of the paper.

Acknowledgements We thank F. S. Katz for assistance with FPLC; R. Kaufman for adeno-ATF4; C. Adams and S. Bullard for Atf $4^{\mid l / f l}$ mice; and A. Ferrante, S. Ramakrishnan, J. Weitz and T. McGraw for discussions. E.C. was supported by NIH grant 5P30CA013696-42. I.T. was funded by grants from the NIH (HL087123 and HL075662) and by a grant from the Merck Investigator Studies Program. L.O. was funded by the NIH grant DK106045 and a grant from the Columbia University Diabetes Research Center (P30 DK063608). Y.S., S.M.N. and M.P.C. were funded by NIH grant DK103047. M.B. was funded by the Deutsche Forschungsgemeinschaft grant SFB1052.

Author Contributions D.S.G., L.O. and I.T. designed the study, analysed data and wrote the manuscript. D.S.G., L.O. and Z.Z. conducted the experiments. S.M.N., Y.S. and M.P.C. made the glucan-encapsulated siRNA particles (GERPs) and helped design these experiments and analyse the data. E.C. conducted the LC-MS/MS studies and assisted with data analysis. M.B. helped with interpretation of data.

Author Information Reprints and permissions information is available at www.nature.com/reprints. The authors declare no competing interests. Readers are welcome to comment on the online version of the paper. Publisher's note: Springer Nature remains neutral with regard to jurisdictional claims in published maps and institutional affiliations. Correspondence and requests for materials should be addressed to L.O. (lo2192@columbia.edu) or I.T. (iat1@columbia.edu).

Reviewer Information Nature thanks P. Scherer and the other anonymous reviewer(s) for their contribution to the peer review of this work. 


\section{METHODS}

Reagents and antibodies. Recombinant mouse and human DPP4 (catalogue no. 954-SE and 9168-SE, respectively) and anti-DPP4 antibody (AF954) were from R\&D Systems. The PAR2 inhibitor GB83 was from Axon Medchem (1622). Recombinant FX (233282) and FXa (233526), and the MEK inhibitor PD98059 (513000) were from Millipore. The FXa inhibitor rivaroxaban (S3002) was from Selleckchem. IRAK1-4 inhibitor I (A3505) and sitagliptin phosphate monohydrate (A4036) were from ApexBio. Tri reagent (T9424), sodium palmitate (P9767), collagenase (C2139), liberase (5401020001), insulin (I9278), the DPP4 inhibitor KR-62436 hydrate (K4264), the caveolin-1 inhibitor daidzein (D7802), PS-1145, IKK inhibitor (P6624), the TAK1 MAPKKK inhibitor 5Z-7-oxozeaenol (O9890), anti-phospho-Thr209-IRAK1 (SAB4504246), and anti- $\beta$-actin antibody (A3854) were from Sigma. Anti-phospho-S473-AKT (4060), anti-AKT (4691), antiphospho-Thr202/Tyr204-ERK1/2 (8544), anti-ERK (9194), anti-phospho-S536-

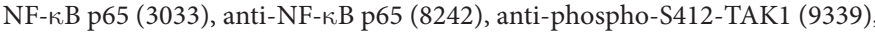
anti-TAK1 (5206), anti-phospho-S338-cRAF1 (9427), anti-cRAF1 (53745) and anti-caveolin-1 (3267) antibodies were from Cell Signaling. Anti-PAR2 antibody (817201) was from Biolegend. The DIO high-fat diet ( $60 \% \mathrm{kcal}$ from fat) (D12492) was from Research Diets. Adenoviral Atf4 (adeno-Atf4) was a gift from R. J. Kaufman (Sanford-Burnham Medical Research Institute), adenoviral Dpp4 (adeno-Dpp4) was purchased from Vector Biolabs, and adeno-associated virus 8 (AAV8) containing either hepatocyte-specific TBG-Cre recombinase (AAV8TBG-cre) or the control vector (AAV8-TBG-lacZ) were purchased from the Penn Vector Core. All adenoviruses were amplified by Viraquest. The AAV8-H1shRNA construct targeting murine DPP 4 was made by annealing complementary oligonucleotides and then ligating them into the pAAV-RSV-GFPH1 vector, as described previously ${ }^{32}$. The resultant constructs were amplified by Salk Institute Gene Transfer, Targeting, and Therapeutics Core. siRNA sequences against murine Par 2 were purchased from GE Dharmacon and siRNA sequences against mouse Cav1 were purchased from Integrated DNA Technologies.

Mouse experiments. Camk $2 g^{f l / f l}$ mice were generated as previously described ${ }^{10}$ and crossed onto the C57BL6/J background. Atf $4^{f l f l}$ mice were generated as previously described ${ }^{33}$. Male Camk $2 g^{f l / f l}$ or Atf $4^{f l / f l}$ mice were fed a high-fat, high-calorie diet (HFD, $60 \% \mathrm{kcal}$ from fat) for 13 weeks starting at three weeks of age and were maintained on a 12-h light-dark cycle. Fifteen-week-old high-fat-diet-fed obese mice (DIO) that had been fed with HFD for nine weeks, four-week old chow-fed $o b / o b$ mice, or sixteen-week-old chow-fed wild-type C57BL/6J mice were purchased from Jackson Laboratories and maintained for one week in the animal facility before commencement of experiments. Sixteen-week-old DIO Camk $2 g^{f l / f l}$ mice that had been fed with HFD for 13 weeks were treated with adeno-associated viruses (AAV) for expression of either hepatocyte-specific TBG-cre ${ }^{34}$ or TBG-lac Z (the control vector) for three weeks to obtain $\mathrm{H}$-CaMKII $(\mathrm{KO})$ and wild-type mice. To obtain ATF4-restored H-CaMKII(KO) or DPP4-restored H-CaMKII(KO) mice, TBG-cre-treated Camk2 $g^{f l f l}$ mice received either adeno-Atf4 (H-CaMKII(KO) plus adeno-Atf4) or adeno-DPP4 (H-CaMKII(KO) plus adeno-Dpp4), while the remaining mice received adeno-lacZ. Similarly, sixteen-week-old Atf $4^{f l / f l}$ DIO mice that had been fed with HFD for 13 weeks were treated with AAV8-TBG-cre or AAV8-TBG-lacZ to obtain hepatocyte-ATF4 KO (H-ATF4(KO)) and wild-type mice. Sixteen-week-old wild-type DIO mice, four-week old $o b / o b$ and sixteenweek-old wild-type C57BL/6J mice were treated with AAV8-H1-shDpp4 or AAV8-H1-control to obtain hepatocyte-specific DPP4 silencing (H-shDpp4) or control mice, respectively. In all mouse experiments, recombinant adenovirus $\left(1 \times 10^{9}\right.$ plaque-forming units per mouse $)$ or adeno-associated virus $\left(1 \times 10^{11}\right.$ genome copies per mouse) was delivered by tail vein injection, and experiments were commenced after 7-28 days or, for the experiment in Extended Data Fig. $6 \mathrm{i}$, after 11 weeks. DIO mice were treated with oral rivaroxaban $(2 \mathrm{mg} / \mathrm{kg})$ twice daily for 20 days. DIO mice were treated for four or seven weeks with sitagliptin by adding it to the drinking water at $0.3 \mathrm{mg} / \mathrm{ml}$, which results in a dose of $\sim 30-45 \mathrm{mg} /$ $\mathrm{kg} /$ day. Mouse plasma samples were collected from lean or obese mice after $4-5 \mathrm{~h}$ of food withdrawal, with free access to water. Blood glucose was measured after 4-5h of food withdrawal in mice using a glucose meter (One Touch Ultra, Lifescan). Plasma insulin levels were measured using the Ultrasensitive Mouse Insulin ELISA Kit (Crystal Chem, 90080) and plasma MCP1, IL6 and TNF $\alpha$ levels were measured using mouse ELISA kits from RayBiotech (ELM-MCP1-1; ELM-IL6-1; ELM-TNFo-1). For gastric inhibitory polypeptide (GIP) and glucagon-like peptide-1 (GLP-1) measurements, mice were fasted overnight and then fed for $4-5 \mathrm{~h}$ before collecting plasma samples into a tube containing DPP4 inhibitor $(5 \mu \mathrm{M})$. Plasma active GIP was measured using Sandwich mouse active GIP (1-42) ELISA Kit (Crystal Chem, 81511) and GLP-1 was assayed in plasma samples using mouse active GLP-1 (7-36) ELISA kit (Eagle Biosciences, GP121-K01). Plasma non-esterified fatty acids (NEFA) were assayed using an enzymatic kit from Wako Diagnostics (HR Series NEFA-HR(2)-Colour Reagent A, 999-34691; Solvent A, 995-34791;
Colour Reagent B, 991-34891; Solvent B, 993-35191; and NEFA standard solution, 276-76491). Intraperitoneal-glucose tolerance tests were performed by intraperitoneal injection of glucose $(1 \mathrm{~g} / \mathrm{kg}$ body weight for DIO, $0.5 \mathrm{~g} / \mathrm{kg}$ body weight for $o b / o b$ and $2 \mathrm{~g} / \mathrm{kg}$ body weight for lean mice) following an overnight fast. Oral glucose tolerance tests were conducted by delivering a $2-\mathrm{g} / \mathrm{kg}$ glucose bolus orally via gavage to DIO mice after a 5 - $\mathrm{h}$ fast. Insulin tolerance tests were performed in mice that were fasted for $4-5 \mathrm{~h}$, by intraperitoneal insulin injection $(0.6 \mathrm{IU} / \mathrm{kg}$ body weight for DIO, $2 \mathrm{IU} / \mathrm{kg}$ body weight for $o b / o b$ and $0.5 \mathrm{IU} / \mathrm{kg}$ body weight for lean mice) and by assaying blood glucose at various time points. Previous studies and pilot experiments formed the basis of power calculations for the various studies. Depending on the experiment, calculations indicated that 3-12 mice per group would enable the testing of our hypotheses based on an expected $25-30 \%$ coefficient of variation and an $80 \%$ chance of detecting a 33\% difference in the key specified endpoints $(P<0.05)$. For all experiments, male mice of the same age and similar weight were randomly assigned to experimental and control groups. On occasion, we analysed a subset of mice for a particular parameter, and the subset was chosen randomly from a full cohort. Pre-specified exclusion criteria were weight loss $10 \%$ of initial body weight or signs of illness or injury requiring euthanasia. According to these pre-specified criteria, the maximum number of mice removed before analysis was three, but was more typically between zero and two. Animal studies were conducted in accordance with the Columbia University Animal Research Committee. Moreover, because switching mice from group caging to single caging, improper handling and placing mice in a restrainer affect stress-sensitive metabolic parameters including plasma DPP4 levels and adipose tissue inflammation (D.S.G., L.O. and I.T., unpublished observations), all experi mental mice were housed in groups to avoid stress from isolation and handled with extra care to avoid stress during blood glucose measurement and blood collection. F4/80 immunostaining of VAT. VAT samples were fixed in $10 \%(\mathrm{v} / \mathrm{v})$ formalin solution for $24 \mathrm{~h}$, followed by incubation with $70 \%$ ethanol $(\mathrm{v} / \mathrm{v})$ for $24 \mathrm{~h}$ and then embedding in paraffin. Six-micrometre sections were mounted on charged glass slides, incubated at $60^{\circ} \mathrm{C}$ for $20 \mathrm{~min}$, and deparaffinized using three washes with xylene. Antigen retrieval was carried out by treating the sections with proteinase $\mathrm{K}(1: 1,000)$ (Invitrogen, AM2546) at $37^{\circ} \mathrm{C}$ for $25 \mathrm{~min}$. The sections were then rinsed and blocked using $5 \%$ serum, followed by incubation overnight at $4{ }^{\circ} \mathrm{C}$ with anti-F4/80 biotin-conjugated primary antibody (1:100) (Miltenyi Biotec, 130101-893). The sections were then incubated with streptavidin-HRP secondary antibody (1:200) (BD Pharmingen, 554066) and then developed in chromagen substrate 3,3'-diaminobenzidine (Cell Signaling, 8059) and counterstained with haematoxylin. For each mouse, 5-10 sections were analysed by microscopic imaging at $20 \times$ magnification. The total number of nuclei and the number of nuclei of F4/80-immunostained cells surrounding adipocytes in CLS were counted for each field. The data are expressed as mean percentage of CLS macrophages per total adipose tissue nuclei.

Portal vein insulin infusion and protein extraction from tissues. After $4-5 \mathrm{~h}$ of food withdrawal, mice were anaesthetized and insulin $(0.6 \mathrm{IU} / \mathrm{kg}$ body weight for DIO, $2 \mathrm{IU} / \mathrm{kg}$ body weight for $o b / o b$ and $0.5 \mathrm{IU} / \mathrm{kg}$ body weight for lean mice) or PBS was injected through the portal vein. Three minutes after injection, liver and adipose tissue were removed, frozen in liquid nitrogen, and kept at $-80^{\circ} \mathrm{C}$ until processing. For protein extraction, tissue samples were cut into small pieces and transferred to tubes containing ice-cold RIPA buffer supplemented with Halt Protease and Phosphatase Inhibitor Cocktail (Thermo Scientific, 78440). Tissue segments were then homogenized on ice, lysates were centrifuged, and the supernatant fractions were used for immunoblot analysis.

Primary hepatocyte experiments. Wild-type C57BL/6J mice (8-10 weeks old) were injected with either control vector or AAV8-shDpp4 (H-shDpp4) via tail vein injection. Ten days after injection, primary mouse hepatocytes were isolated as previously described ${ }^{35}$. Hepatocytes were incubated with either BSA control or palmitate $(50 \mu \mathrm{M})$ for $10 \mathrm{~h}$, with the last $5 \mathrm{~h}$ in serum-free medium. The cells were then treated with $100 \mathrm{nM}$ insulin or vehicle control for $5 \mathrm{~min}$ and immediately frozen in liquid nitrogen and kept at $-80^{\circ} \mathrm{C}$ until further processing. Cells were lysed using $2 \times$ Laemmli buffer supplemented with Halt Protease and Phosphatase Inhibitor Cocktail and lysates were used for immunoblotting.

Preparation of stromal vascular fraction cells and isolation of adipose tissue macrophages. SVF from lean or obese mice was prepared as previously described $^{36}$. In brief, VAT was isolated in ice-cold PBS containing liberase $(50 \mu \mathrm{g} /$ $\mathrm{ml}$ ) and minced into small segments. VAT was digested at $37^{\circ} \mathrm{C}$ for $1 \mathrm{~h}$ with intermittent mixing. After digestion, the solution was centrifuged, buoyant adipocyte were removed, and the cell pellet was retrieved as SVF. SVF cells were then cultured in medium containing $10 \%$ (vol/vol) FBS or plasma from lean or obese mice. For isolation of ATMs, anti-F4/80 biotin-conjugated primary antibody (Miltenyi Biotec, 130-101-893) was added at a 1:10 dilution to $4 \times 10^{6}$ SVF cells in $200 \mu \mathrm{l}$ of PBS containing $5 \%$ of FBS (PBS-FBS), followed by incubation at $4{ }^{\circ} \mathrm{C}$ for $20 \mathrm{~min}$. 
The SVF cells were pelleted by centrifugation, washed once with $1 \mathrm{ml}$ of buffer, and resuspended in $200 \mu \mathrm{l}$ of PBS-FBS. The cells were then incubated with streptavidin-conjugated magnetic microbeads (1:10) (Miltenyi Biotec, 130-048-101) at $4{ }^{\circ} \mathrm{C}$ for $20 \mathrm{~min}$. The cells were rinsed once with PBS-FBS, and ATMs were isolated using magnetic separation columns (Miltenyi Biotec, 130-042-201) and cultured in medium containing $10 \%(\mathrm{vol} / \mathrm{vol}) \mathrm{FBS}$.

Immunoblotting. Proteins from tissue or cell lysates were resolved on 4-20\% Tris-glycine gradient gels and then transferred to nitrocellulose membranes. The membranes were blocked using either $5 \%$ non-fat milk or $5 \%$ BSA followed by incubation with primary antibodies overnight. Blots were then washed thoroughly and probed with HRP-conjugated secondary antibodies for $1 \mathrm{~h}$. Protein bands were detected with Supersignal West Pico enhanced chemiluminescent solution (Thermo Scientific, Cat. No. 34080) and analysed by ImageJ.

Quantitative reverse transcription-PCR. Total RNA was extracted from tissues or primary cell cultures using Tri-reagent (Sigma, T9424). cDNA was synthesized from $2 \mu \mathrm{g}$ total RNA using oligo (dT) and Superscript II (Invitrogen, AM5730G and 18064014). Real time quantitative (q)PCR was performed with a 7500 RealTime PCR System (Applied Biosystems) using SYBR Green master mix (Qiagen, 204145) and gene-specific primers. The sequences of gene-specific primers used for the qPCR assays are described in Supplementary Table 1.

DPP-4 immunodepletion from plasma. DPP-4 was immunodepleted from obese mouse plasma using bead-immobilized anti-DPP-4 antibody (R\&D Systems, AF954) as previously described ${ }^{37}$. In brief, $10 \mu \mathrm{g}$ of anti-DPP- 4 antibody was conjugated with $1.5 \mathrm{mg}$ of Protein-G Dynabeads (Invitrogen, 10004D) in $200 \mu \mathrm{l}$ of binding buffer. After immobilization of anti-DPP4 antibody to beads, $40 \mu \mathrm{l}$ of DIO mouse plasma and $60 \mu \mathrm{l}$ of PBS were mixed with $40 \mu \mathrm{l}$ of antibody-coated bead suspension. The resultant mixture was incubated for $2.5 \mathrm{~h}$ at $4{ }^{\circ} \mathrm{C}$ on a rotating wheel. After $2.5 \mathrm{~h}$, Dynabeads were separated with a magnet and discarded. This was followed by one more round of immunocapture of DPP4 and discarding of beads. DPP-4 proteolytic activity assay was performed on immunodepleted plasma to confirm DPP-4 immunodepletion. For some experiments, DIO mouse plasma was incubated with anti-DPP4-conjugated or control IgG-conjugated beads, followed by elution of the bound material using $50 \mathrm{mM}$ glycine buffer, $\mathrm{pH} 2.8$.

Separation of plasma proteins using FPLC. Lean or obese mouse plasma $(0.2 \mathrm{ml})$ was diluted 1:1 with PBS and injected onto a Superdex 200 Increase 10/300GL FPLC column (GE Healthcare, 28990944). Plasma proteins were eluted from the column at a constant flow of $0.3 \mathrm{ml} / \mathrm{min}$ with PBS (pH 7.4). The effluent was collected in $0.25-\mathrm{ml}$ fractions. The collected fractions were frozen in dry ice and kept at $-80^{\circ} \mathrm{C}$ until further processing.

In-gel protein digestion. Inflammatory obese mouse plasma, DPP4-depleted obese mouse plasma fractions or DPP4 immunoprecipitated from obese mouse plasma (IP-DPP4) were resolved on a 4-20\% Tris-glycine gradient gel and then stained with GelCode Blue Safe Coomassie stain (Thermo Scientific, 24594) for $2 \mathrm{~h}$ followed by de-staining with double distilled water overnight. The resolved proteins were excised and processed at Herbert Irving Comprehensive Cancer Center Proteomics Shared Resource, Columbia University. The excised gel pieces were rehydrated and digested in $80 \mu \mathrm{l}$ of $12.5 \mathrm{ng} / \mu \mathrm{l}$ Trypsin Gold in $50 \mathrm{mM}$ ammonium bicarbonate at $37^{\circ} \mathrm{C}$ overnight. After digestion was complete, condensed evaporated water was collected from the tube walls by brief centrifugation using a benchtop microcentrifuge (Eppendorf). The gel pieces and digestion reaction were mixed with $50 \mu \mathrm{L} 2.5 \%$ trifluoroacetic acid (TFA) and rigorously mixed for $15 \mathrm{~min}$. The solution with extracted peptides was transferred into a fresh tube. The remaining peptides were extracted with $80 \mu \mathrm{l} 70 \%$ acetonitrile-5\% TFA mixture using rigorous mixing for $15 \mathrm{~min}$. The extracts were pooled and dried to completion $(1.5-2 \mathrm{~h})$ in a SpeedVac. The dried peptides were reconstituted in $30 \mu \mathrm{l} 0.1 \%$ TFA by mixing for $5 \mathrm{~min}$ and stored in ice or at $-20^{\circ} \mathrm{C}$ before analysis.

LC-MS/MS analysis. The concentrated peptide mix was reconstituted in a solution containing $2 \%$ acetonitrile and $2 \%$ formic acid for mass spectrometry analysis. Peptides were eluted from the column using a Dionex Ultimate 3000 Nano liquid chromatography system with a 10 -min gradient from $2 \%$ buffer B to $35 \%$ buffer B ( $100 \%$ acetonitrile, $0.1 \%$ formic acid). The gradient was switched from $35 \%$ to $85 \%$ buffer $\mathrm{B}$ over $1 \mathrm{~min}$ and held constant for $2 \mathrm{~min}$. Finally, the gradient was changed from $85 \%$ buffer B to $98 \%$ buffer A ( $100 \%$ water, $0.1 \%$ formic acid) over $1 \mathrm{~min}$, and then held constant at $98 \%$ buffer A for a further $5 \mathrm{~min}$. The application of a $2.0-\mathrm{kV}$ distal voltage electrosprayed the eluting peptides directly into the Thermo Fusion Tribrid mass spectrometer equipped with an EASY-Spray source (Thermo Scientific). Mass spectrometer-scanning functions and HPLC gradients were controlled by the Xcalibur data system (Thermo Finnigan).

Database search and interpretation of MS/MS data. Tandem mass spectra from raw files were searched against a mouse protein database using the Proteome Discoverer 1.4 software (Thermo Finnigan). The Proteome Discoverer application extracts relevant MS/MS spectra from the .raw file and determines the precursor charge state and the quality of the fragmentation spectrum. The Proteome
Discoverer probability-based scoring system rates the relevance of the best matches found by the SEQUEST algorithm. The mouse protein database was downloaded as FASTA-formatted sequences from the Uniprot protein database (database released in September, 2014). The peptide mass search tolerance was set to 10 p.p.m. A minimum sequence length of seven amino acids residues was required. Only fully tryptic peptides were considered. To calculate confidence levels and false discovery rates (FDR), Proteome Discoverer generates a decoy database containing reverse sequences of the non-decoy protein database and performs the search against this concatenated database (non-decoy + decoy). Scaffold (Proteome Software) was used to visualize searched results. The discriminant score was set at less than $1 \%$ FDR, determined on the basis of the number of accepted decoy database peptides, to generate protein lists for this study. Spectral counts were used for estimation of relative protein abundance between samples.

Mouse liver nuclei preparation and chromatin immunoprecipitation assays. Mouse liver tissues were homogenized using a Dounce homogenizer (Wheaton) with the loose pestle in 1:10 (w:v) of ice-cold NP-40 lysis buffer supplemented with protease inhibitor cocktail. Liberation of nuclei was monitored by DAPI staining and fluorescence microscopy. To purify intact nuclei, lysates were then layered over a sucrose gradient consisting of $1 \mathrm{M}$ sucrose overlaid with $0.68 \mathrm{M}$ sucrose and then centrifuged at 4000 r.p.m. for $20 \mathrm{~min}$ at $4{ }^{\circ} \mathrm{C}$. Following a washing step nuclear pellets were cross-linked with $1 \%$ fresh formaldehyde in PBS for $10 \mathrm{~min}$ at room temperature. Cross-linking was terminated by addition of $200 \mathrm{mM}$ Tris$\mathrm{HCl}(\mathrm{pH} 9.4)$ and $1 \mathrm{mM} \mathrm{DTT}$ for $10 \mathrm{~min}$ followed by centrifugation at 2,500 r.p.m. for $10 \mathrm{~min}$ at $4{ }^{\circ} \mathrm{C}$. Nuclear pellets were suspended in SDS lysis buffer containing protease inhibitors, incubated for $10 \mathrm{~min}$ on ice, and DNA was sheared in a cold water bath in a focused-ultrasonicator (Covaris, S2) to obtain DNA fragments with an average size of $500 \mathrm{bp}$. Fragmented chromatin was pre-cleaned by incubating with normal rabbit IgG (Santa Cruz, sc-2025) for $1 \mathrm{~h}$ at $4^{\circ} \mathrm{C}$, followed by $1 \mathrm{~h}$ of incubation with $50 \mu \mathrm{l}$ protein $\mathrm{G}$ magnetic beads (Pierce) at $4^{\circ} \mathrm{C}$ with rotation. A rabbit anti-ATF4 antibody (Cell Signaling, 11815) to pull down ATF4-binding complexes, and a control rabbit anti-haemagglutinin antibody (Santa Cruz) were used to pull down non-specific binding complexes. Immunoprecipitated chromatin fragments were reverse cross-linked, digested by proteinase $\mathrm{K}$, and purified using QIAquick PCR Purification Kit (Qiagen, 28104). The presence of ATF4 in the Dpp4 exonic region was quantified by qPCR and expressed relative to the input genomic DNA. The sequences of primers used for the ChIP-qPCR assays are described in Supplementary Table 2 .

Macrophage assays. ATMs, peritoneal macrophages or BMDMs were incubated for $4 \mathrm{~h}$ in medium containing $10 \%$ plasma from lean or obese mice and then assayed for Mcpl or Il6 mRNA using quantitative reverse transcription-PCR (RT-qPCR). ATMs, bone marrow-derived macrophages or human macrophages were incubated for $4 \mathrm{~h}$ with $0.25 \mathrm{U} / \mathrm{ml} \mathrm{rFXa,} 175 \mathrm{ng} / \mathrm{ml} \mathrm{rDPP} 4$, or both protein together. The cells were then assayed for Mcp1 or Il6 mRNA using RT-qPCR.

Glucan-encapsulated siRNA particle (GERP) study in $o b / o b$ mice. FITC-labelled glucan shells (GS) containing non-targeting (control) siRNA or siRNAs agains Par2 or Cav1 were prepared and loaded as previously described ${ }^{38,39}$. In brief, $2.4 \mathrm{mg}$ of FITC-labelled glucan shells-lectin, $7.2 \mathrm{nmol}$ of siRNA, and $120 \mathrm{nmol}$ of endoporter were combined to form FITC-GERPs. Five-week old $o b / o b$ mice received 12 daily doses of FITC-GERPs by intraperitoneal injection, which contained $0.2 \mathrm{mg}$ FITC-GS, $0.6 \mathrm{nmol}$ siRNA and $10 \mathrm{nmol}$ endoporter. Glucose tolerance and insulin tolerance tests were performed on days seven and ten of GERP treatment, respectively. Mice were euthanized $24 \mathrm{~h}$ after the last injection.

Statistical analysis. All results are presented as mean \pm s.e.m. For experiments with two groups, $P$ values were calculated using the two-tailed Student's $t$-test for normally distributed data and the Mann-Whitney rank sum test for nonnormally distributed data. For experiments with more than two groups, $P$ values were calculated using one-way or two-way ANOVA for normally distributed data and the Kruskal-Wallis by ranks test for non-normally distributed data, with Student-Newman-Keuls or Dunn's post hoc test. Error bars that appear to be absent from certain graph symbols signify s.e.m. values smaller than the graph symbols.

Data availability. The data that support the findings of this study are available from the corresponding author upon reasonable request.

32. Lisowski, L. et al. Selection and evaluation of clinically relevant AAV variants in a xenograft liver model. Nature 506, 382-386 (2014).

33. Ebert, S. M. et al. Stress-induced skeletal muscle Gadd45a expression reprograms myonuclei and causes muscle atrophy. J. Biol. Chem. 287 27290-27301 (2012)

34. Mu, X. et al. Hepatocellular carcinoma originates from hepatocytes and not from the progenitor/biliary compartment. J. Clin. Invest. 125, 3891-3903 (2015).

35. Shimano, H. et al. Overproduction of cholesterol and fatty acids causes massive liver enlargement in transgenic mice expressing truncated SREBP-1a. J. Clin. Invest. 98, 1575-1584 (1996). 


\section{RESEARCH LETTER}

36. Orr, J. S., Kennedy, A. J. \& Hasty, A. H. Isolation of adipose tissue immune cells. J. Vis. Exp. 22, e50707 (2013)

37. Nazarian, A. et al. Inhibition of circulating dipeptidyl peptidase 4 activity in patients with metastatic prostate cancer. Mol. Cell. Proteomics 13, 3082-3096 (2014).
38. Aouadi, M. et al. Orally delivered siRNA targeting macrophage Map4k4 suppresses systemic inflammation. Nature 458, 1180-1184 (2009).

39. Cohen, J. L. et al. Peptide- and amine-modified glucan particles for the delivery of therapeutic siRNA. Mol. Pharm. 13, 964-978 (2016). 


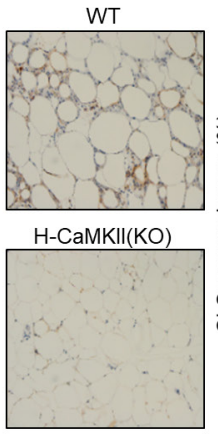

d

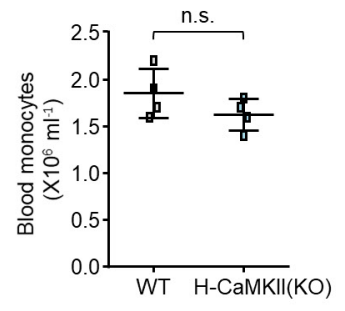

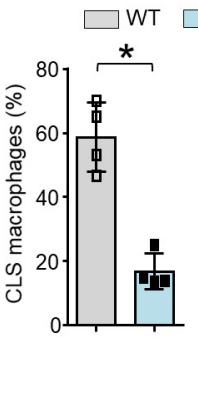

b $\square$ WT

$\square$ H-CaMKII(KO)

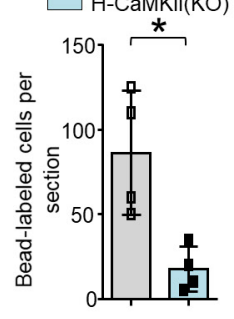

C

$\square$ WT $\square$ H-CaMKII(KO)

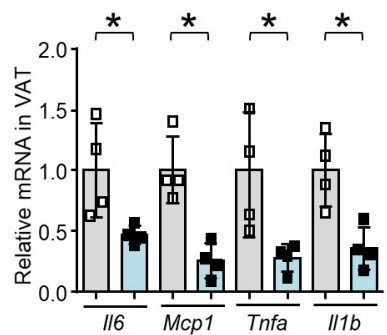

f

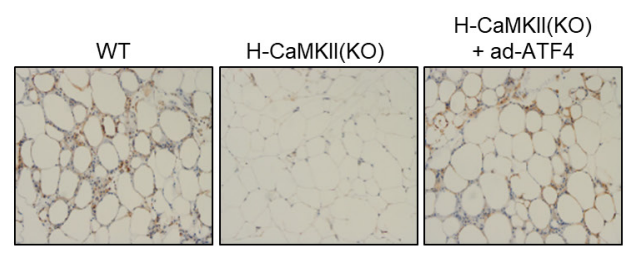

e $\square$ WT $\square$ H-CaMKII(KO)
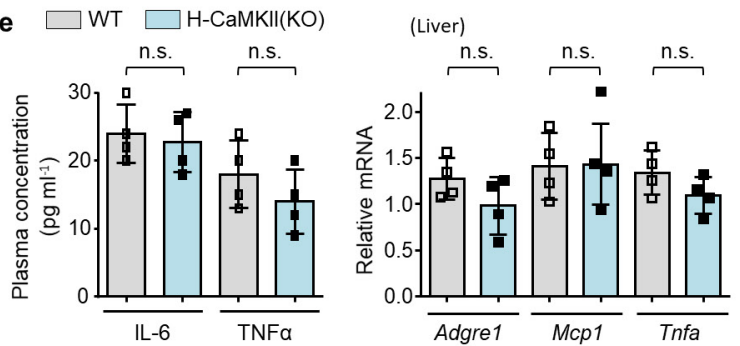

g
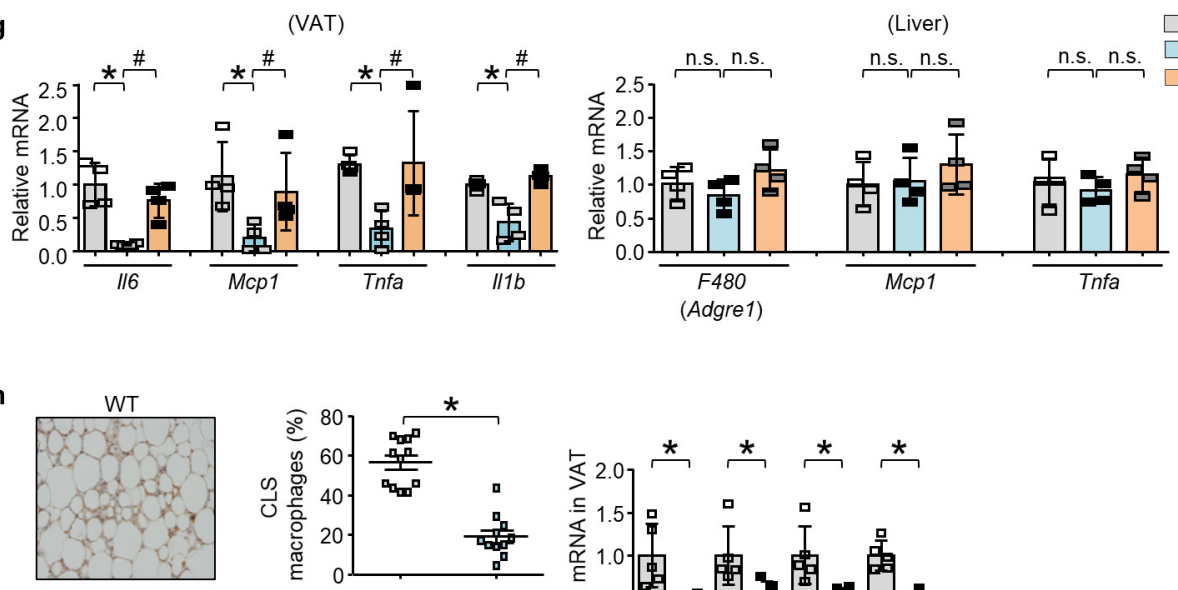

H-ATF4 (KO)
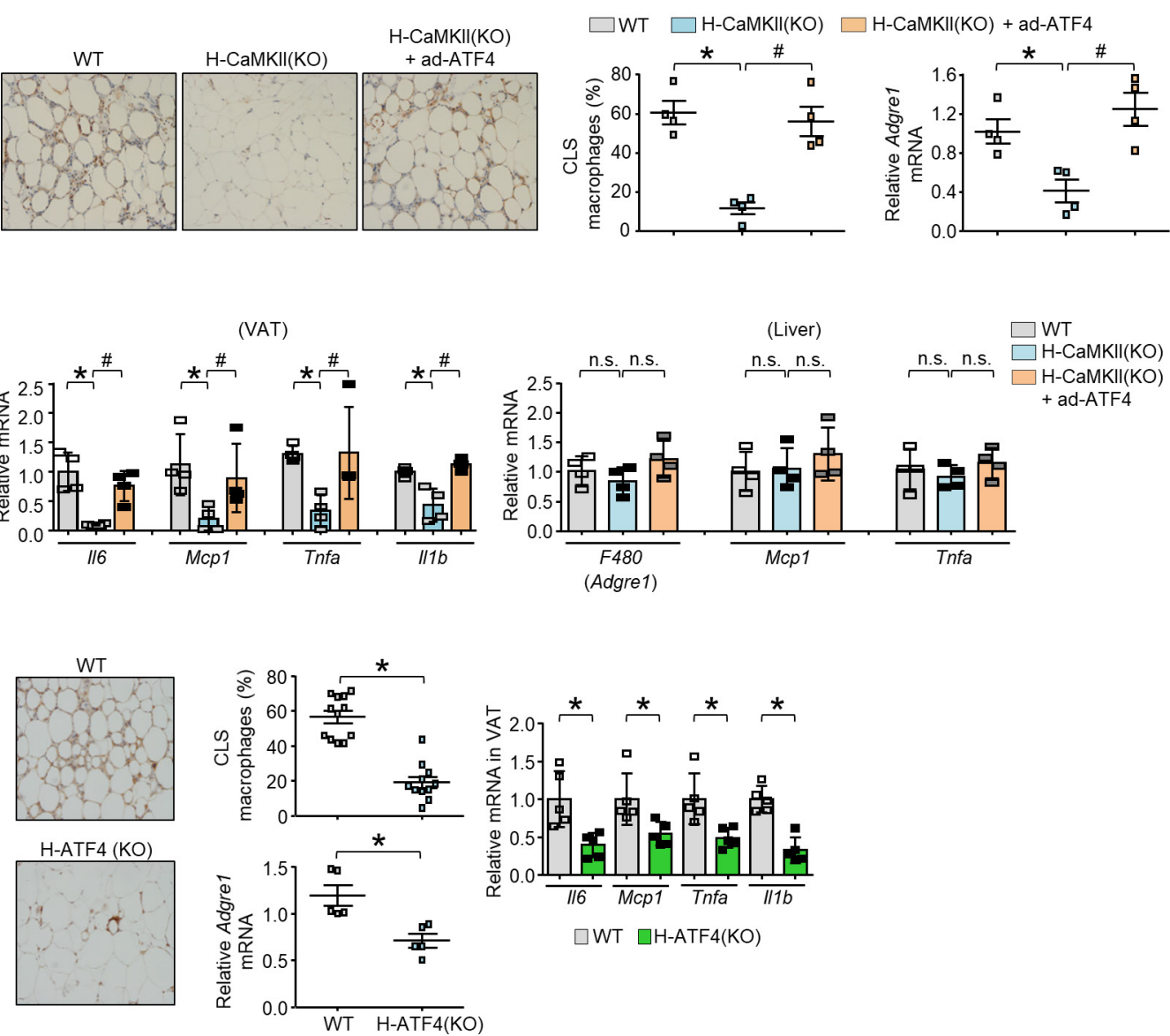

Extended Data Figure 1 | See next page for caption. 


\section{RESEARCH LETTER}

Extended Data Figure 1 Hepatocyte-specific deletion of CaMKII or ATF4 in DIO mice lowers VAT inflammation. a-c, Sixteen-week-old Camk $2 g^{f l f l}$ mice that had been previously fed the DIO diet for 13 weeks were injected intravenously with AAV8-TBG-cre (H-CaMKII(KO)) or AAV8-TBG-lacZ (wild-type, WT). Mice were analysed after three additional weeks on the DIO diet. a, Representative images of VAT immunostained for F4/80, with quantification of crown-like-structure (CLS) macrophages, and expression of Adgre1 mRNA, which encodes $\mathrm{F} 4 / 80$. b, As in a, except that the mice were injected with fluorescent beads using a procedure that labels circulating Ly $6 \mathrm{c}^{\mathrm{hi}}$ monocytes, and then bead-labelled cells were assayed in VAT sections. c, mRNAs for Il6, $M c p 1, T n f$ and $I l 1 b$ in VAT. d, Blood monocyte count. e, Plasma IL6 and TNF $\alpha$ measured by ELISA, and quantification of Adgre 1, Mcp 1 and Tnfa mRNA in liver. In a-e, $n=4$ mice per group; mean \pm s.e.m.; $* P<0.05$; n.s., not significant by two-tailed Student's $t$-test. f, g, Mice similar to those in $\mathbf{a}-\mathbf{c}$, and from a third group in which Camk $2 g^{f l / f l}$ mice were injected intravenously with adeno-Atf4 and AAV8-TBG-cre (H-CaMKII(KO) + adeno-Atf4).f, CLS macrophages and Adgre 1 mRNA in VAT were quantified, with representative images of F4/80-stained VAT. g, Il6, Mcp1, Tnfa and $I l 1 b$ mRNA in VAT and Adgre 1, Mcp 1 and Tnfa mRNA in liver were quantified. Note that the first two groups of mice received adeno-lac $Z$ instead of adeno-Atf4. $n=4$ mice per group; mean \pm s.e.m.; $* P<0.05$ by one-way ANOVA; n.s., not significant. h, AAV8-TBG-cre (H-ATF4(KO)) or AAV8-TBG-lacZ (wild-type) was injected intravenously into 16-weekold $A f t 4^{f l / f l}$ mice previously fed the DIO diet for 13 weeks. After three further weeks on the DIO diet, VAT from these mice was immunostained for F4/80 to identify macrophages, the percentage of macrophages in CLS was quantified, and the VAT was assayed for Adgre 1 and the indicated inflammatory mRNAs. Twelve wild-type and $11 \mathrm{H}$-ATF4(KO) mice was analysed for CLS macrophages, and a randomly selected subset of five wild-type and five H-ATF4(KO) mice was analysed for the VAT mRNAs. Mean \pm s.e.m., ${ }^{*} P<0.05$ by two-tailed Student's $t$-test. 
a

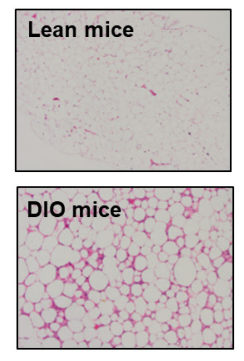

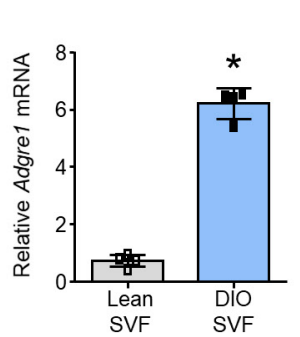

b

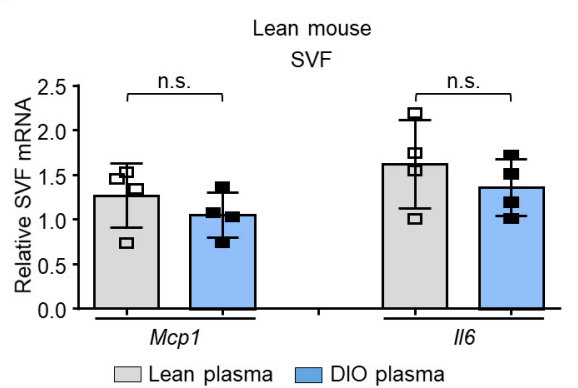

C (ex vivo macrophage assay)
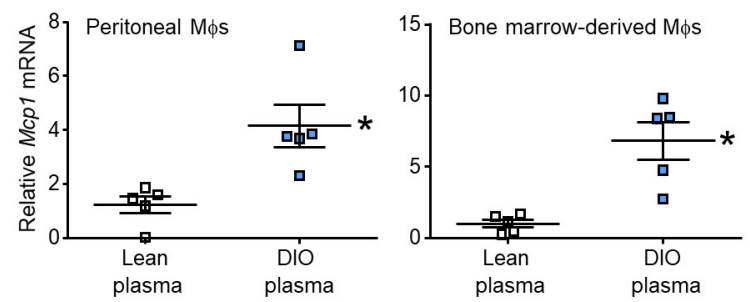

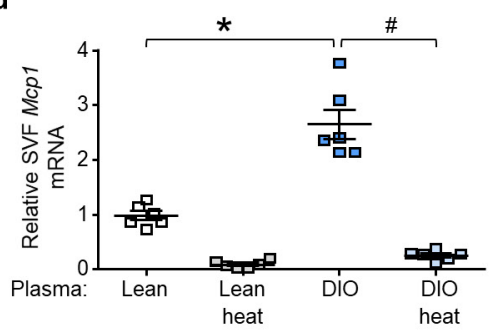

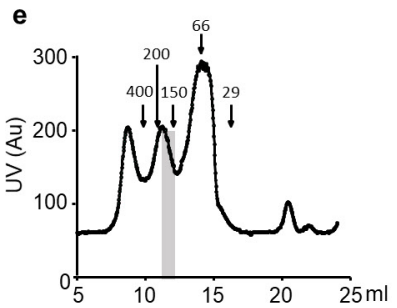
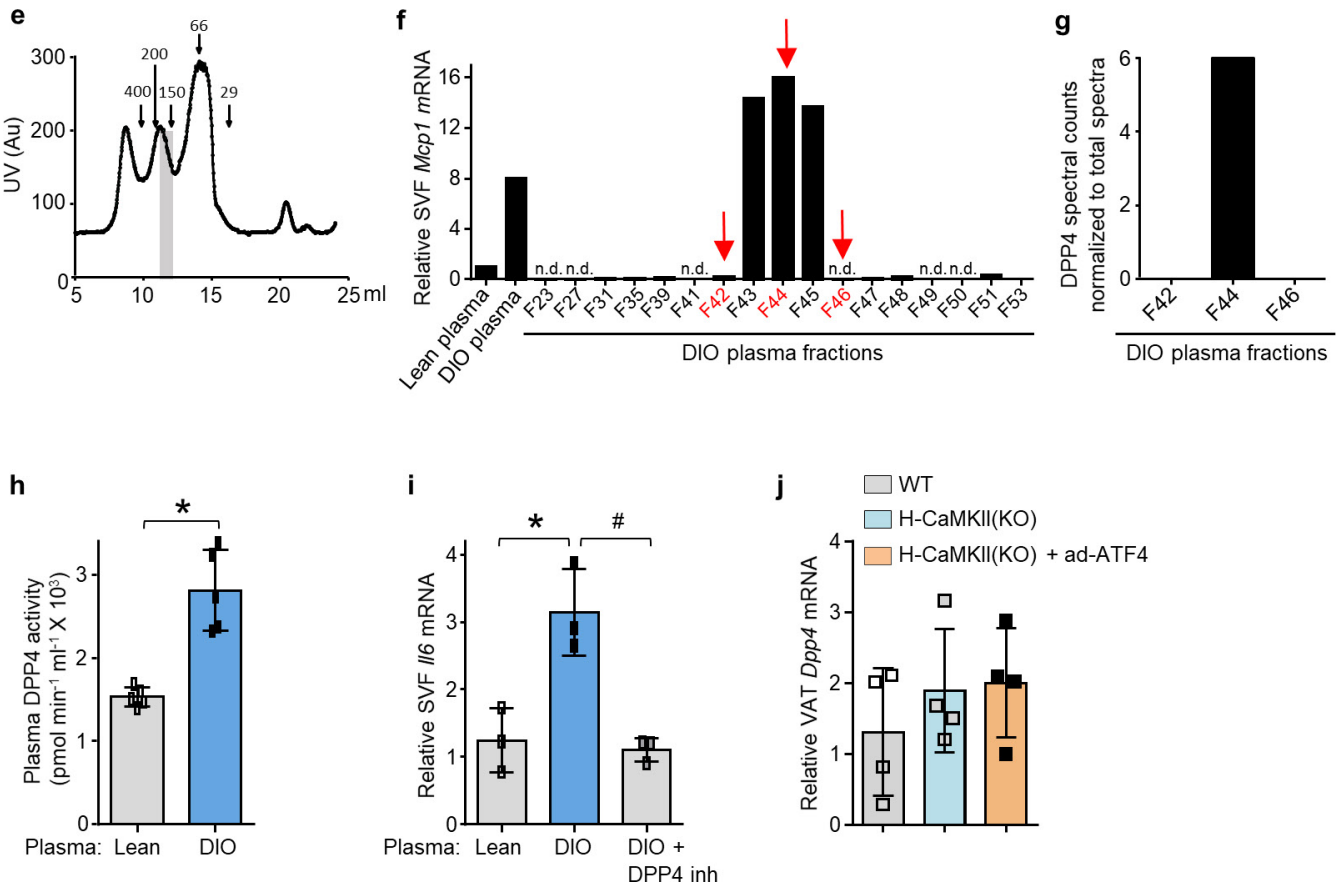

Extended Data Figure $2 \mid$ DPP4 in the plasma of DIO mice induces Mcp1 and Il6 in SVF cells from the VAT of obese mice and in

macrophages. a, Representative images of haemotoxylin and eosin-stained VAT sections from lean and DIO mice and Adgre1 mRNA levels in SVF from lean and DIO mice. $n=4$ mice per group; mean \pm s.e.m.; $* P<0.05$. b, SVF cells from the VAT of lean mice were incubated for $4 \mathrm{~h}$ in medium containing $10 \%(\mathrm{v} / \mathrm{v})$ plasma from lean or DIO mice and then assayed for $M c p 1$ and Il6 mRNA. $n=4$ mice per group; mean \pm s.e.m.; $* P<0.05$; n.s., not significant. c, $M c p 1$ mRNA was assayed in mouse peritoneal macrophages $(\mathrm{M} \phi \mathrm{s})$ or bone marrow-derived macrophages that were incubated for $4 \mathrm{~h}$ with medium containing $10 \%(\mathrm{v} / \mathrm{v})$ plasma from lean or DIO mice. $n=5$ technical replicates per group; mean \pm s.e.m.; $* P<0.05$. d, Mcp1 mRNA levels in SVF cells that were incubated for $4 \mathrm{~h}$ with medium containing $10 \%(\mathrm{v} / \mathrm{v})$ control or heat-inactivated (heat) plasma from the indicated groups of mice. $n=6$ mice per group; mean \pm s.e.m.; $* P<0.05$. e, UV protein chromatogram obtained after

fractionation of DIO mice plasma using gel-filtration FPLC; vertical grey bar depicts peak of activity shown in f. f, Obese mouse SVF cells were incubated with medium containing $10 \%$ lean or DIO mouse plasma or the indicated FPLC fractions from $\mathbf{e}$ and assayed for Mcp1 mRNA. n.d., Mcp1 mRNA not detected. Arrows indicate the fractions that were selected for LC-MS/MS analysis. g, LC-MS/MS normalized spectral counts corresponding to DPP4 in the FPLC fractions from $\mathbf{f}$. $\mathbf{h}$, DPP4 activity in the plasma of lean and DIO mice. $n=5$ mice per group; mean \pm s.e.m.; $* P<0.05$. i, SVF cells from DIO mice were incubated for $4 \mathrm{~h}$ with medium containing $10 \%(\mathrm{v} / \mathrm{v})$ lean or DIO mouse plasma that was pre-treated for $1 \mathrm{~h}$ with or without $10 \mu \mathrm{M}$ DPP4 inhibitor KR62436. The cells were then assayed for Il6 mRNA. $n=3$ technical replicates per group; mean \pm s.e.m.; $* P<0.05$. j, VAT from the mice in Extended Data Fig. 1f, g was assayed for Dpp4 mRNA $(n=4)$. Data in $\mathbf{a}-\mathbf{c}$ and $\mathbf{h}$ were analysed by two-tailed Student's $t$-test; data in $\mathbf{i}$ and $\mathbf{j}$ were analysed by one-way ANOVA $(\mathbf{g}, \mathbf{h})$; data in $\mathbf{d}$ were analysed by two-way ANOVA. 
a

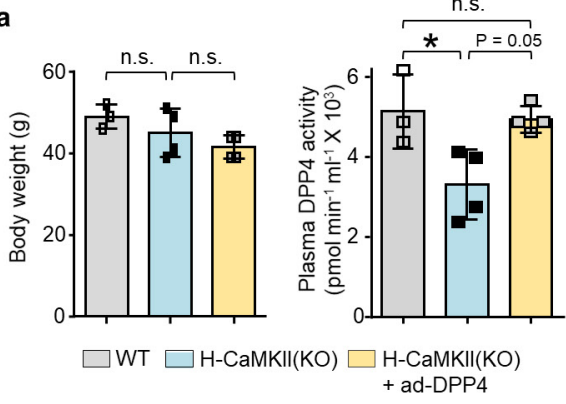

b
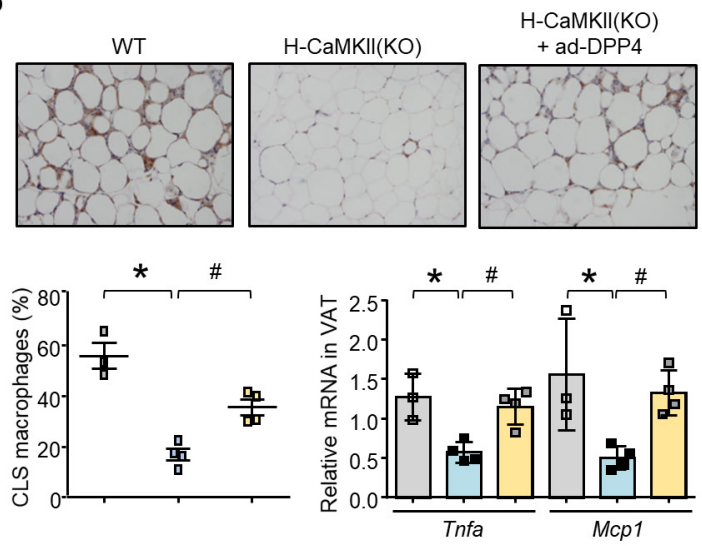

$\square$ WT $\square$ H-CaMKII(KO) $\square$ H-CaMKII(KO) + ad-DPP4

d
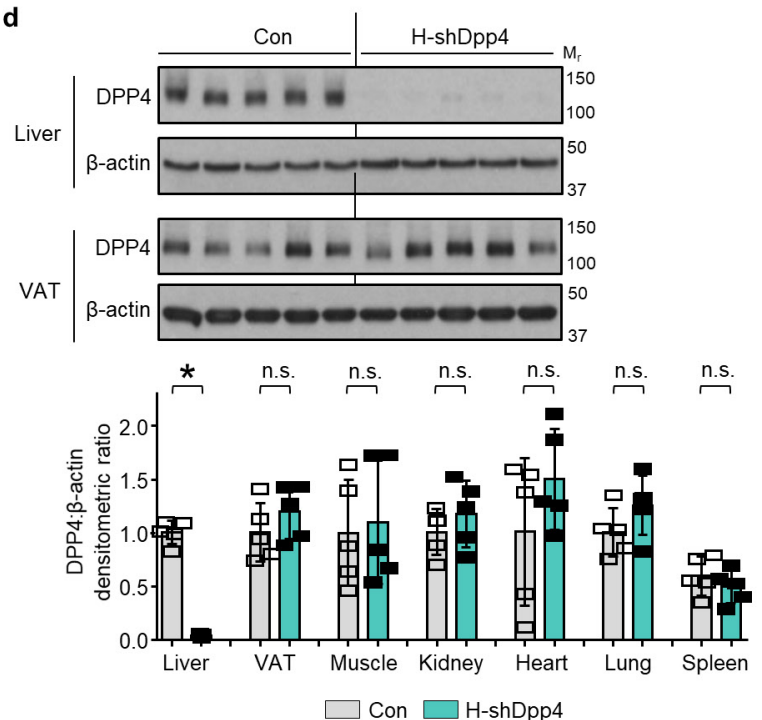
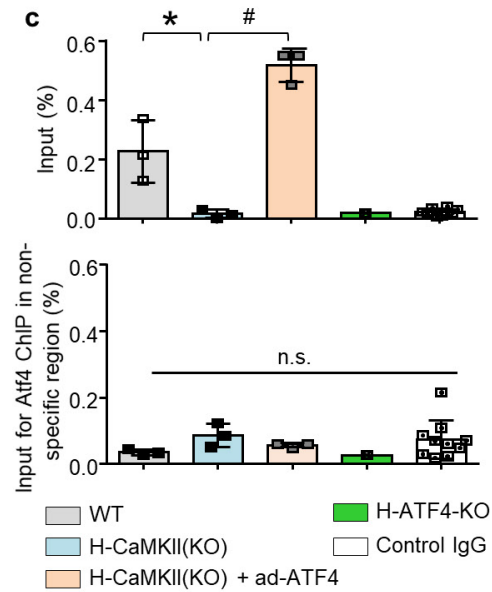

e
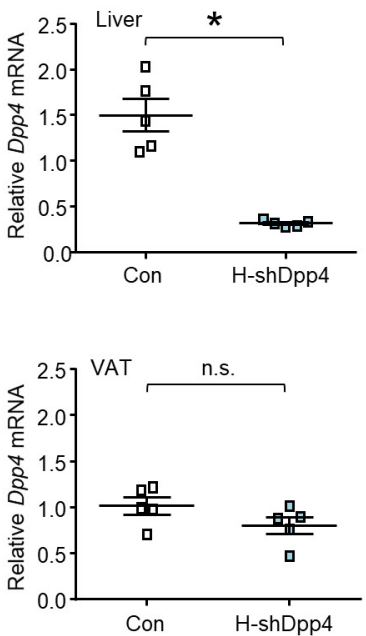

Extended Data Figure 3 Restoration of DPP4 in livers of

$\mathrm{H}$-CaMKII(KO) mice abrogates suppression of VAT inflammation in DIO mice; ATF4 ChIP of the Dpp4 gene; and AAV8-H1-shDpp4 treatment lowers hepatic DPP4. Wild-type and H-CaMKII(KO) mice and a third group in which Camk2 $g^{f l l f l}$ mice were injected intravenously with adeno- $D p p 4$ together with the AAV8-TBG-cre (H-CaMKII(KO) + adeno-Dpp4) were analysed as follows. a, Body weight, plasma DPP4 activity and liver and VAT DPP4 protein. $\mathbf{b}$, Representative images of VAT immunostained with F4/80 antibody, with quantification of CLS macrophages and Tnfa and Mcp1 mRNA in VAT. Note that the first two groups of mice received adeno-lac $Z$ instead of adeno-DPP4. In $\mathbf{a}$ and $\mathbf{b}$, $n=3-4$ mice per group; mean \pm s.e.m.; $* P<0.05$ by one-way ANOVA; n.s., non-significant. For gel source data, see Supplementary Fig. 1. c, Top, ChIP was performed with liver extracts from the indicated groups of mice using anti-ATF4 or control IgG antibodies. The region spanning the predicted ATF4-binding sequence in exon 1 of Dpp 4 was amplified by RT-qPCR and normalized to the values obtained from the input. $n=3$ ChIP assays for wild-type, $\mathrm{H}$-CaMKII(KO), H-CaMKII(KO) + adeno-Atf4; $n=1$ for H-ATF4(KO); $n=10$ for control IgG; mean \pm s.e.m.; $\#, * P<0.05$ by one-way ANOVA for groups $1-3$. Bottom, per cent input for ATF4 ChIP using liver extracts from the indicated DIO mice and PCR primers for a region in the $D p p 4$ gene that does not contain a consensus sequence for ATF4 binding. $n=3 \mathrm{ChIP}$ assays for wild type, $\mathrm{H}$-CaMKII(KO), H-CaMKII(KO) + adeno-Atf4; $n=1$ for H-ATF4(KO); $n=10$ for control IgG; mean \pm s.e.m.; n.s., non-significant by one-way ANOVA. d, e, Sixteen-week-old mice previously fed with the DIO diet for 10 weeks were injected intravenously with hepatocyte-specific AAV8H1-shDpp4 (H-shDpp4) or control AAV8-H1 vector (con). After four additional weeks on DIO diet, the mice were analysed as follows. d, DPP4 immunoblot, with densitometric quantification of DPP4 protein in the indicated tissues; representative of three independent experiments. e, $D p p 4 \mathrm{mRNA}$ in liver and VAT. In $\mathbf{d}$ and $\mathbf{e}, n=5$ mice per group; mean \pm s.e.m.; $* P<0.05$; n.s., non-significant by two-tailed Student's $t$-test. See Supplementary Fig. 1 for gel source data. 
a

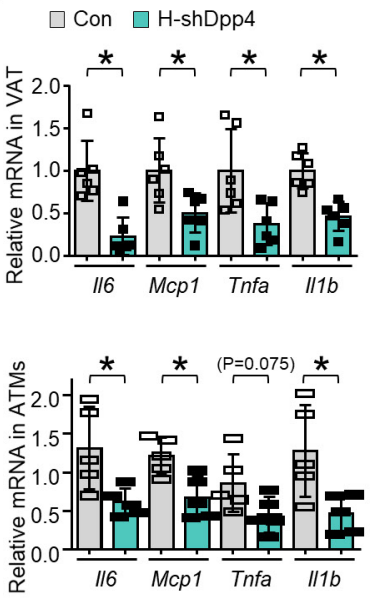

a

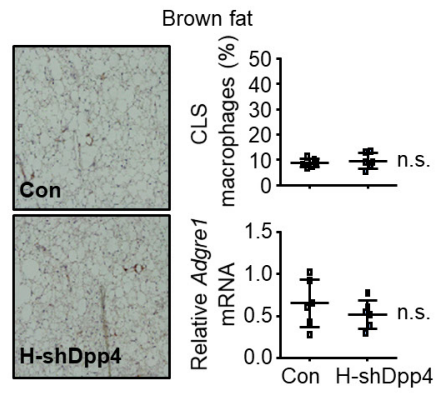

b

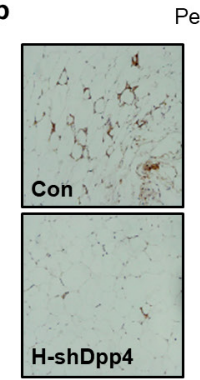

rirenal fat
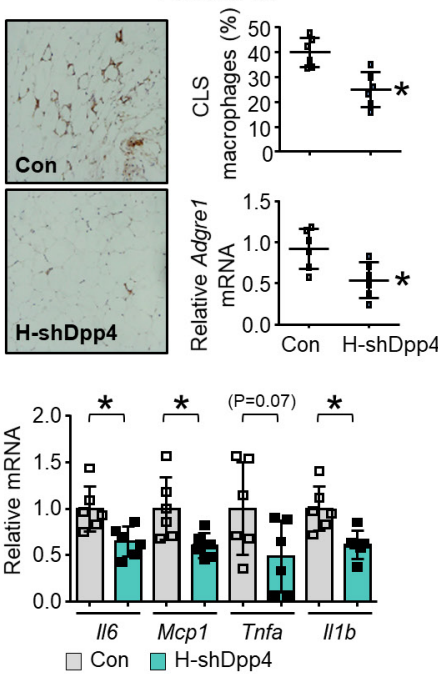

e

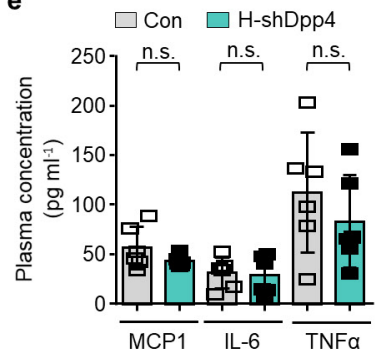

C

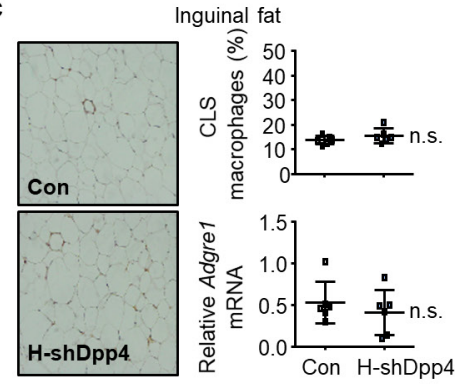

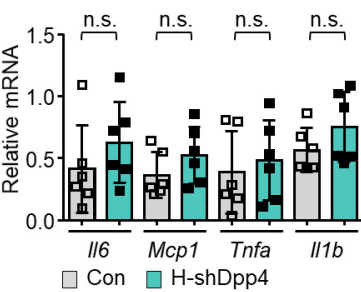

f $\square$ Con $\square \mathrm{H}$-shDpp4

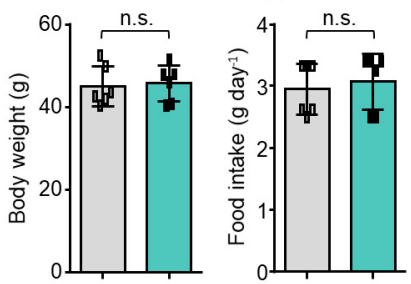

g $\square$ Con $\square$ H-shDpp4
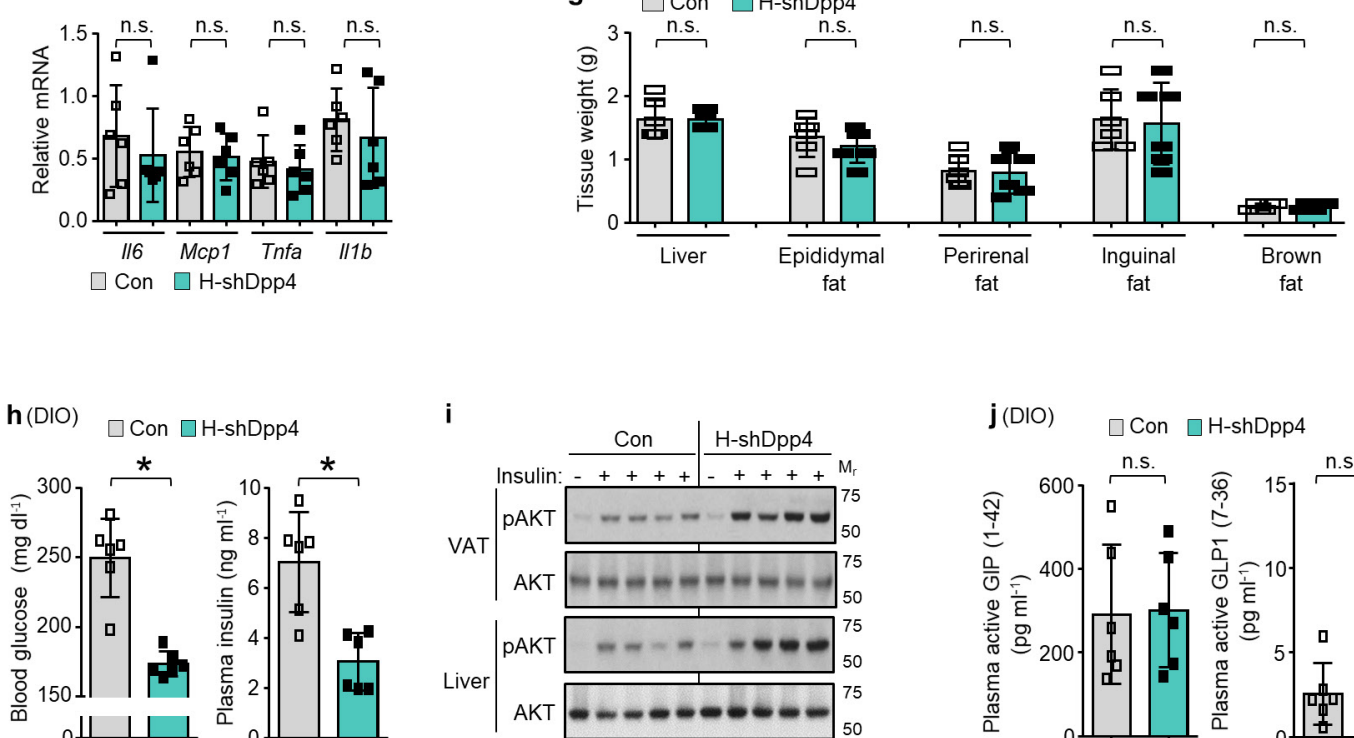

i
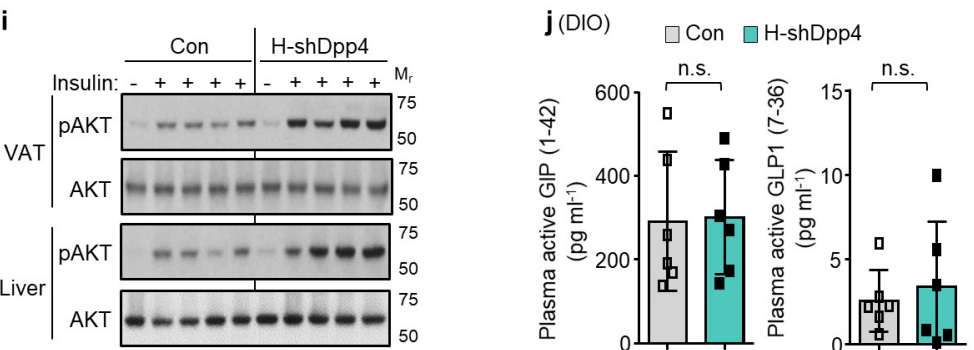

Extended Data Figure 4 Silencing of DPP4 in liver suppresses VAT inflammation and improves metabolism without increasing plasma incretins. a, Top, 16-week-old mice previously fed with the DIO diet for 10 weeks were injected intravenously with AAV8-H1-shDpp4 (H-shDpp4) or control AAV8-H1 vector (con). After four weeks, the mice were assayed for Il6, Mcp1, Tnfa and Ill $b$ mRNA in VAT. Bottom, control and $\mathrm{H}$-shDpp4-treated mice similar to those above were analysed nine days after adenovirus injections for Il6, Mcp 1, Tnfa and Ill b mRNA in ATMs. b-g, H-shDPP4 and control mice were analysed after four weeks. b-d, Representative images of adipose tissue immunostained with F4/80 antibody, with quantification of CLS macrophages, Adgre1 and inflammatory gene mRNA expression in perirenal, inguinal and brown fat. e, Plasma MCP1, IL6 and TNF $\alpha$. f, Body weight and food intake. $\mathbf{g}$, Weights of liver and the indicated adipose tissue depots. In a-g, $n=5-6$ mice per group; mean \pm s.e.m.; $* P<0.05$; n.s., non-significant by twotailed Student's $t$-test. $\mathbf{h}-\mathbf{j}$, Mice similar to those in $\mathbf{b}-\mathbf{g}$ were assayed. $\mathbf{h}$, Blood glucose and plasma insulin. $\mathbf{i}, \mathrm{p}-\mathrm{AKT}$ and total AKT in VAT and liver after insulin injection into the portal vein. $n=1$ PBS-injected, $n=4$ insulin-injected mice per group; blots are representative of three independent experiments. Gel source data are shown in Supplementary Fig. 1. j, Plasma active GIP (1-42) and GLP1 (7-36). In $\mathbf{h}$ and $\mathbf{j}, n=6$ mice per group; mean \pm s.e.m.; $* P<0.05 ;$ n.s., non-significant by two-tailed 

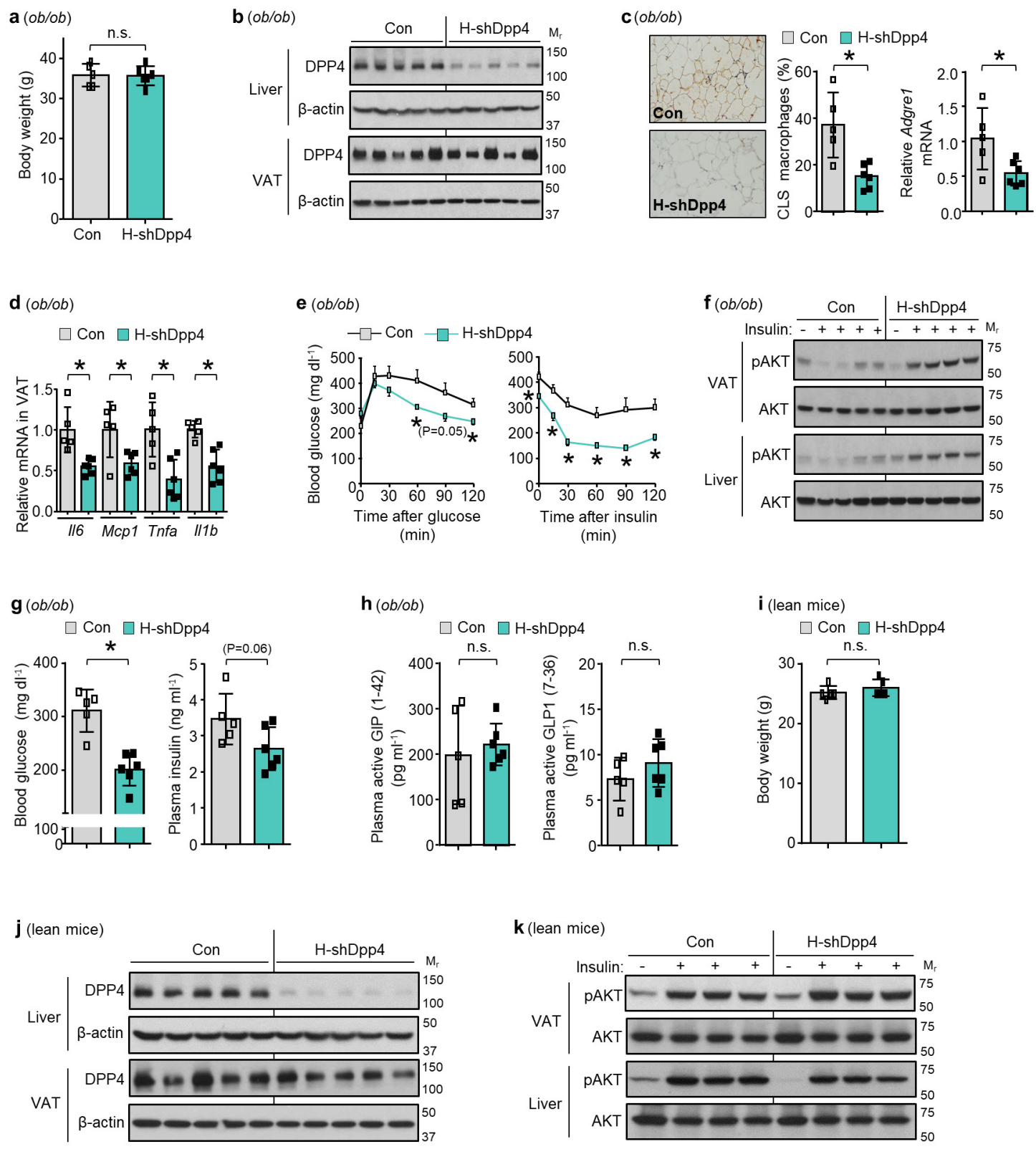

I (lean mice)

$\square$ Con $\square \mathrm{H}$-shDpp4
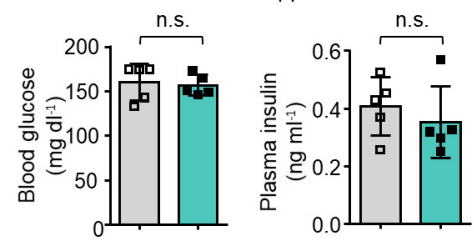

m (lean mice)

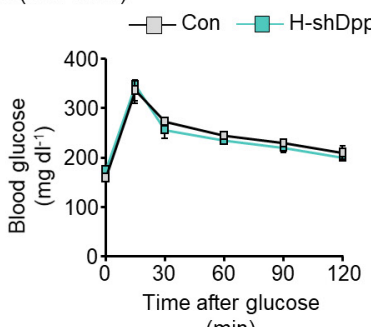

(min)

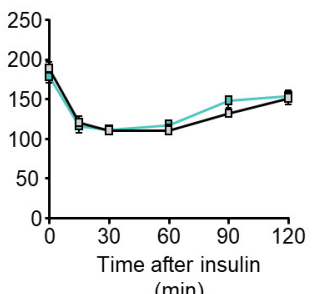

(min)
Extended Data Figure $5 \mid$ Hepatocyte-specific silencing of DPP4 improves glucose metabolism in $o b / o b$ mice without increasing plasma incretins and does not affect VAT inflammation or glucose metabolism in lean mice. a-h, Five-week-old chow-fed $o b / o b$ mice were injected intravenously with AAV8-H1-shDpp4 (H-shDpp4) or AAV8-H1-control (con), and were assayed four weeks later. a, Body weight. $\mathbf{b}$, Immunoblot of liver and VAT DPP4. c, Representative images of VAT immunostained with F4/80 antibody, with quantification of CLS macrophages and Adgre1 mRNA in VAT. d, Il6, $M c p 1$, Tnfa and Il1 b mRNA in VAT. e, Blood glucose after challenge with intraperitoneal glucose or insulin. f, p-AKT and total AKT in VAT and liver extracts after portal vein insulin injection. g, Blood glucose and plasma insulin $5 \mathrm{~h}$ after food withdrawal. h, Plasma active GIP (1-42) and GLP1 (7-36). i-m, Sixteen-week-old chow-fed wild-type lean mice were injected intravenously with AAV8-H1-shDpp4 (H-shDpp4) or AAV8-H1-control (Con) and were analysed as follows. i, Body weight. j, Immunoblot of DPP4 in liver and VAT. $\mathbf{k}, \mathrm{p}$-AKT and total AKT in VAT and liver extracts after portal vein insulin injection. 1, Blood glucose and plasma insulin $5 \mathrm{~h}$ after food withdrawal. $\mathbf{m}$, Blood glucose after challenge with intraperitoneal glucose or insulin. In all panels, $n=5-6$ mice per group; mean \pm s.e.m.; n.s., non-significant by two-tailed Student's $t$-test. For gel source data, see Supplementary Fig. 1. 
$\mathbf{a}$ (hepatocytes from lean mice)

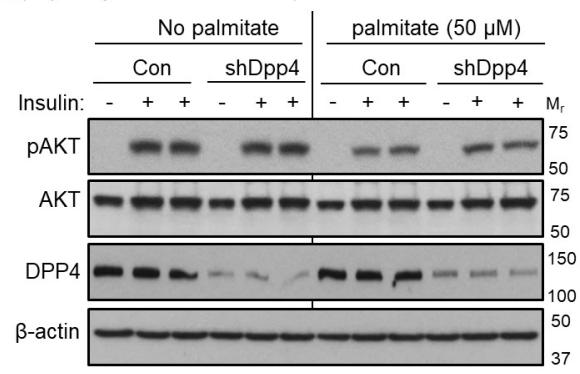

b (plasma)
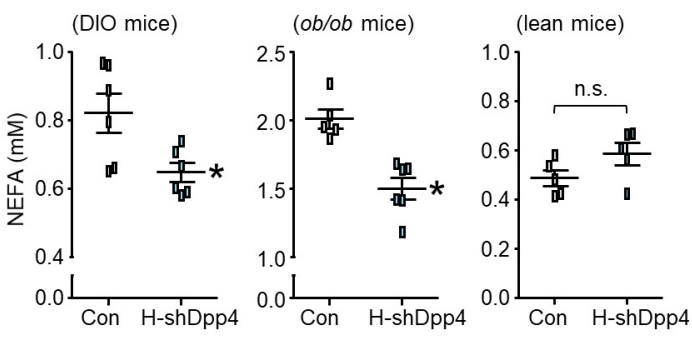

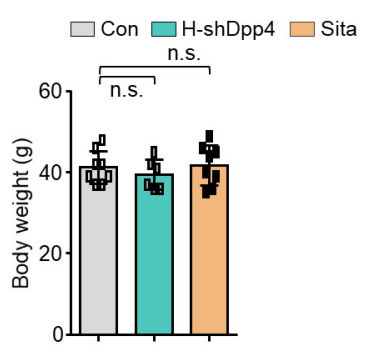

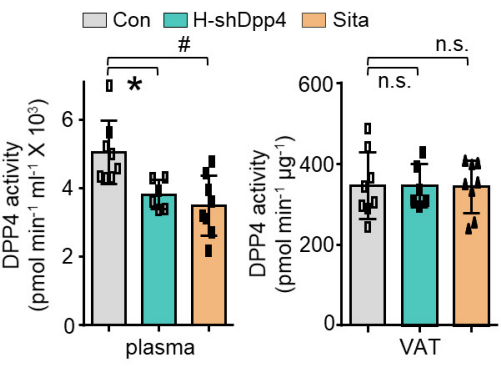

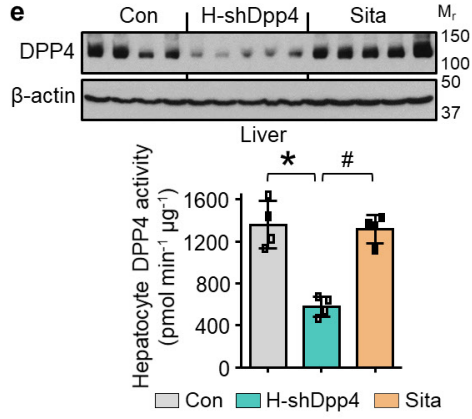

f

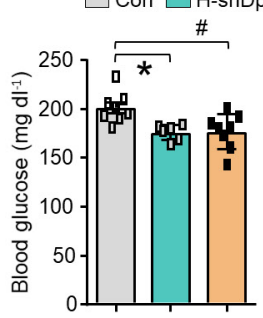

g

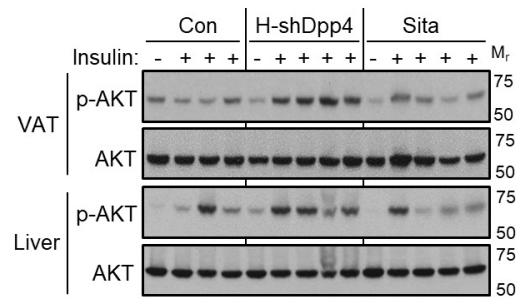

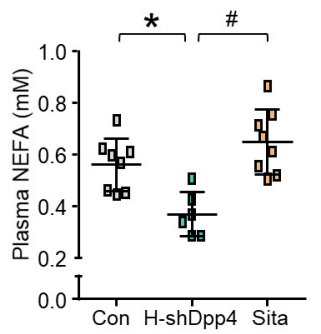

i

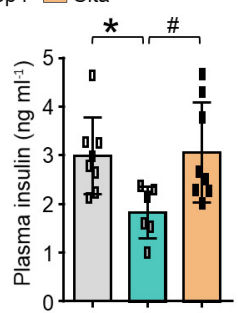

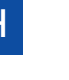


a
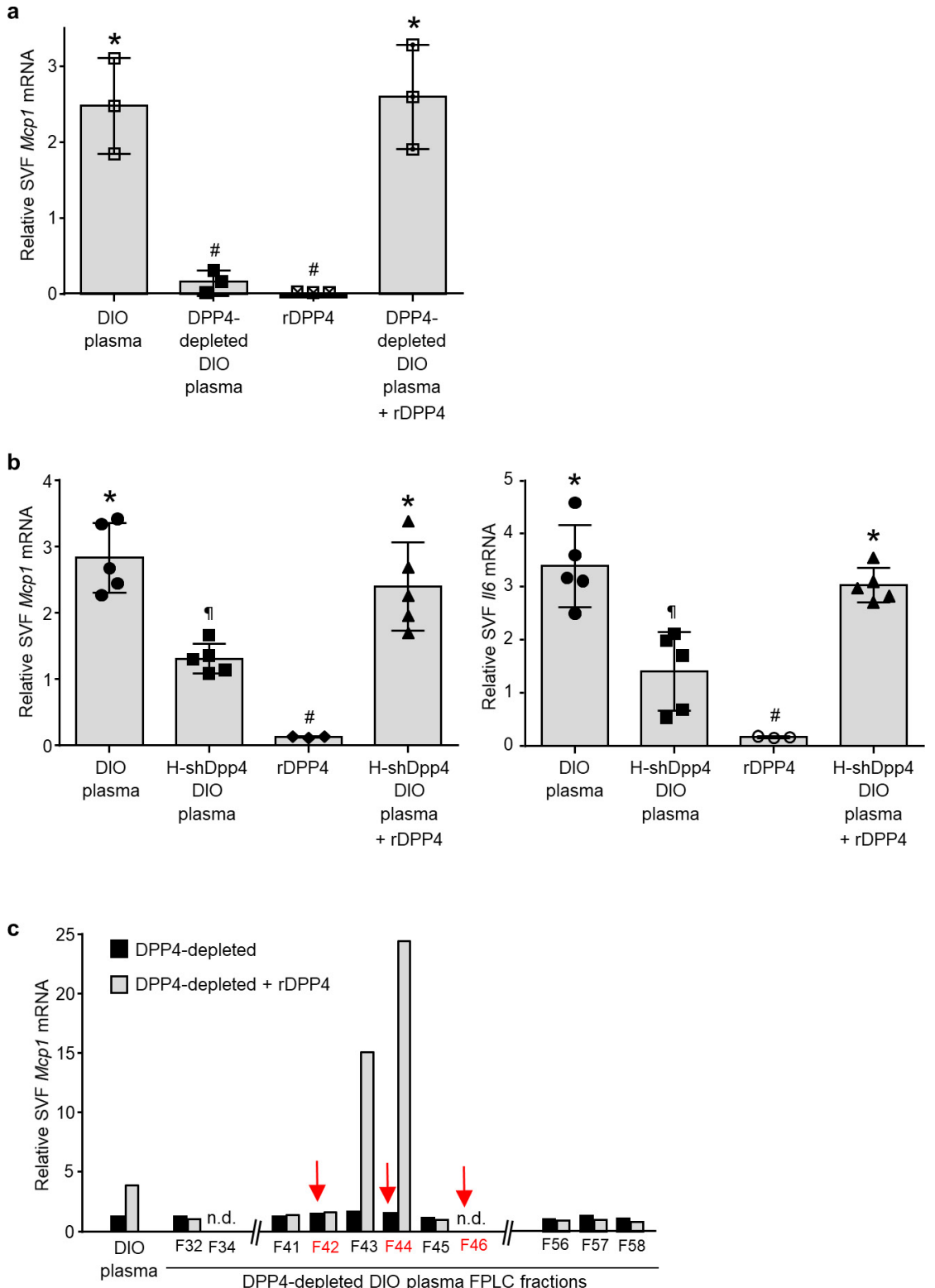

Extended Data Figure 7 | DPP4 requires a plasma factor to induce inflammation in SVF. a, Mcp1 mRNA was assayed in SVF cells that were incubated for $4 \mathrm{~h}$ with medium containing $10 \%(\mathrm{v} / \mathrm{v})$ DIO mouse plasma; DIO mouse plasma immunodepleted of DPP4; recombinant DPP4 (rDPP4) alone; or rDPP4 plus DIO mouse plasma immunodepleted of DPP4. $n=3$ technical replicates per group;. mean \pm s.e.m. b, As a, except plasma from $\mathrm{H}$-shDPP4 DIO mice were used instead of DPP4depleted obese mouse plasma, and both Mcp 1 and Il6 were assayed $(n=5$ technical replicates per group; mean \pm s.e.m.). In $\mathbf{a}$ and $\mathbf{b}$, groups with different symbols are different from each other $(P<0.05$ by one-way ANOVA). c, DPP4-depleted plasma from DIO mice was fractionated by Superdex-200 FPLC. Each fraction, as well as unfractionated DIO mouse plasma, was incubated with SVF cells with or without rDPP4, followed by assay of $M c p 1$ mRNA. n.d., $M c p 1$ mRNA not detected. The fraction numbers in red (arrows) were selected for LC-MS/MS analysis. The data are from a single experiment. 


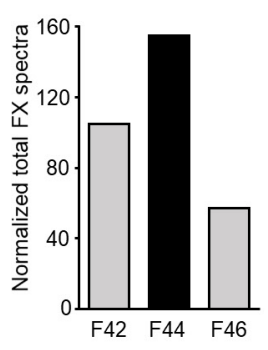

b (SVF)

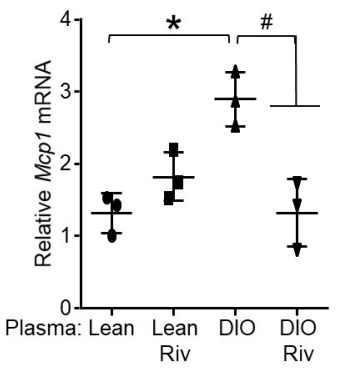

(BMDM)

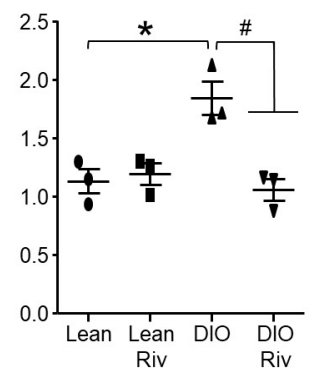

c (BMDM)

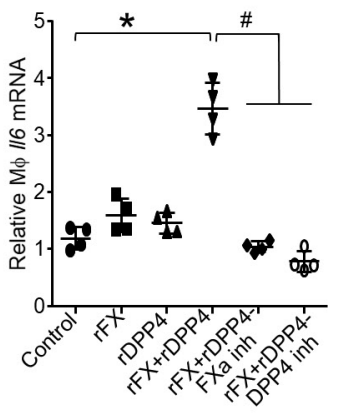

d
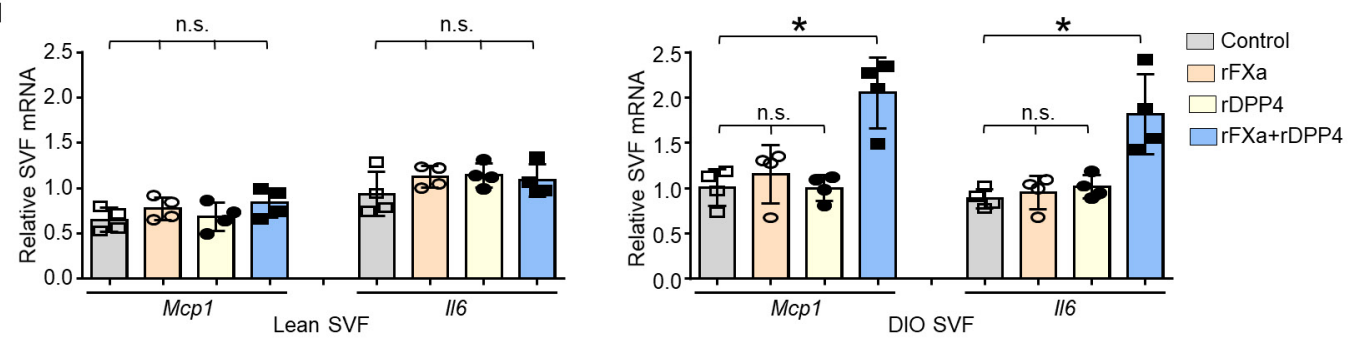

e
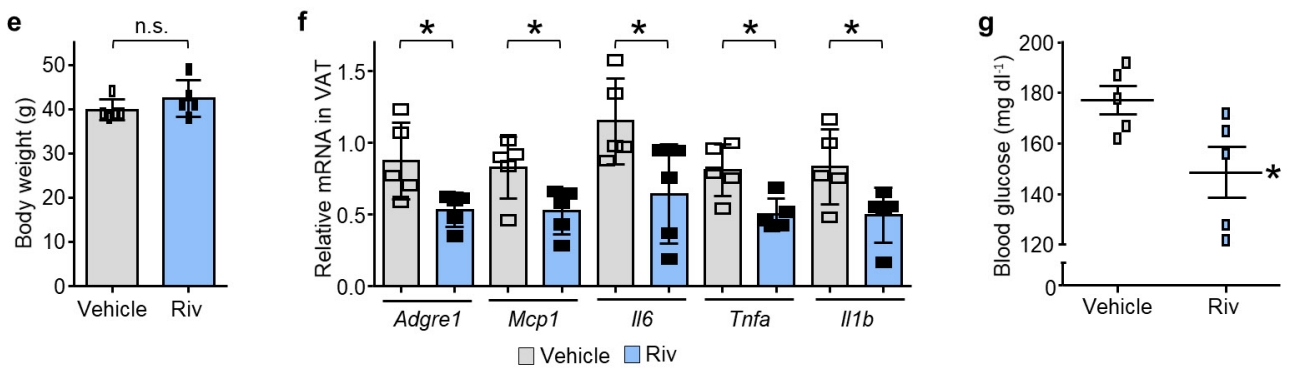

h

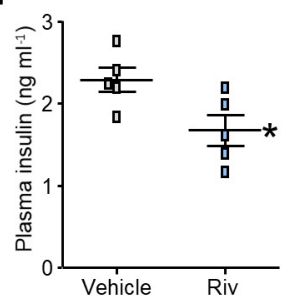

i

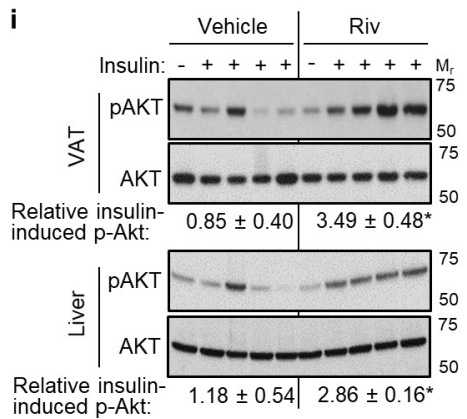

j

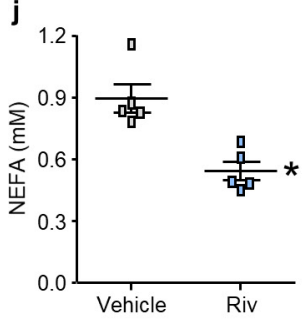

Extended Data Figure 8 | DPP4 and FXa synergistically promote VAT inflammation, and inhibition of FXa improves glucose metabolism. a, LC-MS/MS normalized spectral counts corresponding to FX in the indicated FPLC fractions from Extended Fig. 7c. b, SVF cells or BMDMs were pre-incubated for $1 \mathrm{~h}$ with or without $10 \mu \mathrm{M} \mathrm{FXa}$ inhibitor rivaroxaban (riv) and then incubated with medium containing $10 \%(\mathrm{v} / \mathrm{v})$ plasma from lean or DIO mice and assayed for $M c p 1$ mRNA. $n=3$ technical replicates per group; mean \pm s.e.m.; ${ }^{*} \# P<0.05$ by twoway ANOVA. c, BMDMs were pretreated with or without $10 \mu \mathrm{M}$ FXa inhibitor rivaroxaban (riv) or $10 \mu \mathrm{M}$ DPP4 inhibitor KR62436, followed by incubation for $4 \mathrm{~h}$ with $\mathrm{rFX}$ or $\mathrm{rDPP} 4$ alone or both together. Il6 mRNA was then quantified. $n=4$ technical replicates per group; mean \pm s.e.m.; \#,* $P<0.05$ by one-way ANOVA. d, SVF cells from lean or DIO mice were treated with $\mathrm{rFX}$ or $\mathrm{rDPP} 4$ alone or in combination for $4 \mathrm{~h}$, and then Mcp1 and Il6 mRNA levels were assayed ( $n=4$ technical replicates per group; mean \pm s.e.m.; $* P<0.05$ and n.s., non-significant by one-way ANOVA). $\mathbf{e}-\mathbf{j}$, The control and rivaroxaban-treated mice from Fig. 3b, c were assayed. e, Body weight. f, Adgre 1, Mcp 1, Il6, Tnfa and Illb mRNA in VAT. g, h, Blood glucose and plasma insulin $5 \mathrm{~h}$ after food withdrawal. i, p-AKT and total AKT in VAT and liver after portal vein insulin injection. $\mathbf{j}$, Plasma non-esterified fatty acids (NEFA). In $\mathbf{e}-\mathbf{h}$ and $\mathbf{j}, n=5$ mice per group; mean \pm s.e.m.; $* P<0.05$ and n.s., non-significant by two-tailed Student's $t$-test. In $\mathbf{i}$, the mean fold increases of the plus-insulin values relative to the minus-insulin value, based on the densitometric ratio of p-AKT:total AKT, are shown below the blots $(n=1 \mathrm{PBS}$-injected and $n=4$-insulin injected mice per group; mean \pm s.e.m.; $* P<0.05$ ). For gel source data, see Supplementary Fig. 1. 


\section{RESEARCH LETTER}

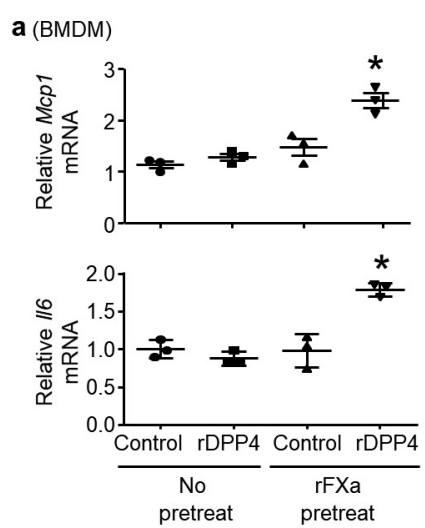

d (human macrophages)

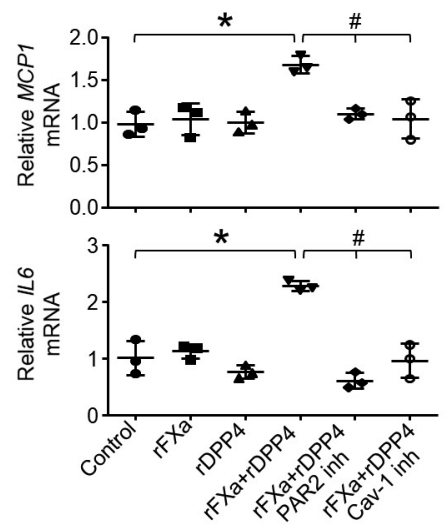

f

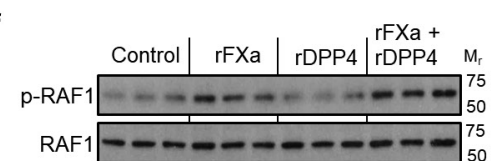

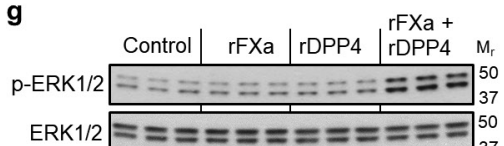
\begin{tabular}{rl|r|r|r|r} 
& Control & rFXa & rDPP4 & rDPP4 & $M_{r}$ \\
\hline P-P6Xa & & & \\
\hline
\end{tabular}
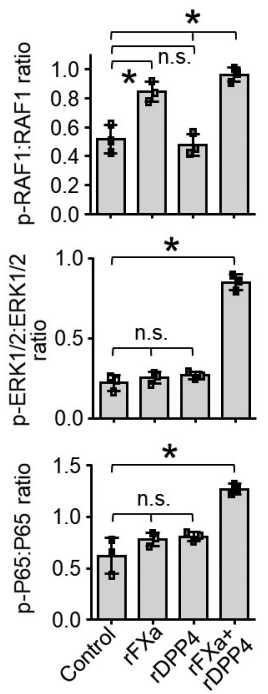
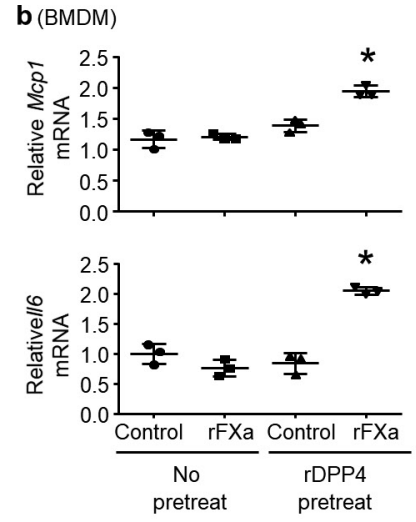

e

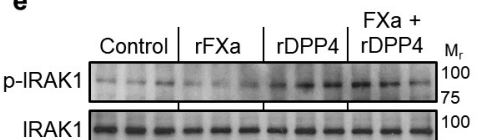

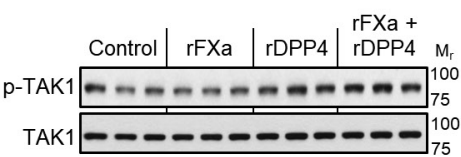

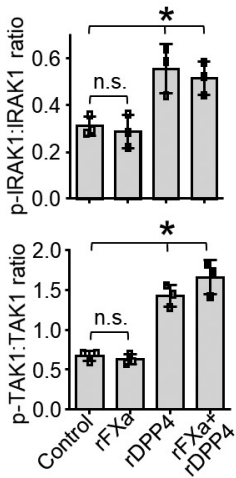

c (ATMs)
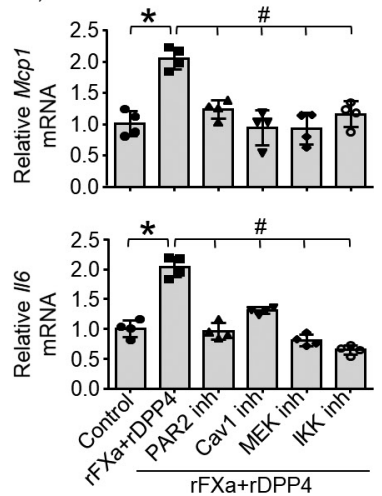
Extended Data Figure 9 | DPP4 and FXa synergistically induce inflammatory signalling pathways in macrophages. a, BMDMs were pretreated for $4 \mathrm{~h}$ with or without $0.25 \mathrm{U} \mathrm{rFXa}$, medium was then removed, cells were washed and treated with $10 \mu \mathrm{M}$ rivaroxaban (riv) to inhibit residual FXa activity. The cells were then incubated for $4 \mathrm{~h}$ with or without $70 \mathrm{ng}$ rDPP 4 and assayed for Mcp1 and Il6 mRNA. b, BMDMs were pretreated for $4 \mathrm{~h}$ with or without $70 \mathrm{ng} \mathrm{rDPP} 4$, medium was then removed, cells were washed and treated with $10 \mu \mathrm{M}$ KR62436 to inhibit residual DPP4 activity. The cells were then incubated for $4 \mathrm{~h}$ with or without $0.25 \mathrm{U}$ rFXa and assayed for Mcp 1 and Il6 mRNA. In a and $\mathbf{b}, n=3$ technical replicates per group; mean \pm s.e.m.; $* P<0.05$ versus all other groups by one-way ANOVA. c, ATMs were pre-treated for $1 \mathrm{~h}$ with or without $10 \mu \mathrm{M}$ of the PAR2 inhibitor GB83, $25 \mu \mathrm{M}$ of the CAV1 inhibitor daidzein, $10 \mu \mathrm{M}$ of the MEK inhibitor PD98059 or $10 \mu \mathrm{M}$ of the IKK inhibitor PS-1145. The cells were then incubated for $4 \mathrm{~h}$ with or without rFXa and rDPP4 and assayed for $M c p 1$ and Il6 mRNA. $n=4$ technical replicates per group; mean \pm s.e.m.; ${ }^{*} \# P<0.05$ by one-way ANOVA. d, Human monocytederived macrophages were pre-treated for $1 \mathrm{~h}$ with or without $10 \mu \mathrm{M}$ PAR2 inhibitor (GB83) or $25 \mu \mathrm{M}$ CAV1 inhibitor (daidzein) and then incubated for $4 \mathrm{~h}$ with or without $\mathrm{rFXa}, \mathrm{rDPP} 4$ or both and assayed for $M C P 1$ and $I L 6$ mRNA. $n=3$ technical replicates; mean \pm s.e.m.; $\#, * P<0.05$ versus all other groups by one-way ANOVA. $\mathbf{e}-\mathbf{g}$, Bone marrow-derived macrophages were incubated for $10 \mathrm{~min}$ with or without $0.25 \mathrm{U} \mathrm{rFXa}, 70 \mathrm{ng} \mathrm{rDPP} 4$ or $\mathrm{rFX}$ a plus $\mathrm{rDPP} 4$ and then assayed by immunoblot for phosphorylated and total IRAK1, TAK1, RAF1, ERK1/2 and P65, followed by densitometric quantification. The data are representative of two $(\mathbf{e}, \mathbf{f})$ or three $(\mathbf{g})$ independent experiments. For all panels, $n=3$ technical replicates per group; mean \pm s.e.m.; $* P<0.05$ versus other groups and n.s., not significant by one-way ANOVA.

$\mathbf{h}-\mathbf{j}$, Bone marrow-derived macrophages were pretreated for $1 \mathrm{~h}$ with or without the following inhibitors. h, IRAK-1/4 inhibitor-I, $0.5 \mu \mathrm{M}$. i, TAK1 MAPKKK inhibitor Z-7-oxozeaenol, $0.1 \mu \mathrm{M}$. j, RAF1 inhibitor GW5074, $1 \mu \mathrm{M}$. Cells were then incubated for $4 \mathrm{~h}$ with or without rFXa plus rDPP4 and assayed for $M c p 1$ and Il6 mRNA. $n=4$ technical replicates per group; mean \pm s.e.m.; $* P<0.05$ by one-way ANOVA. 

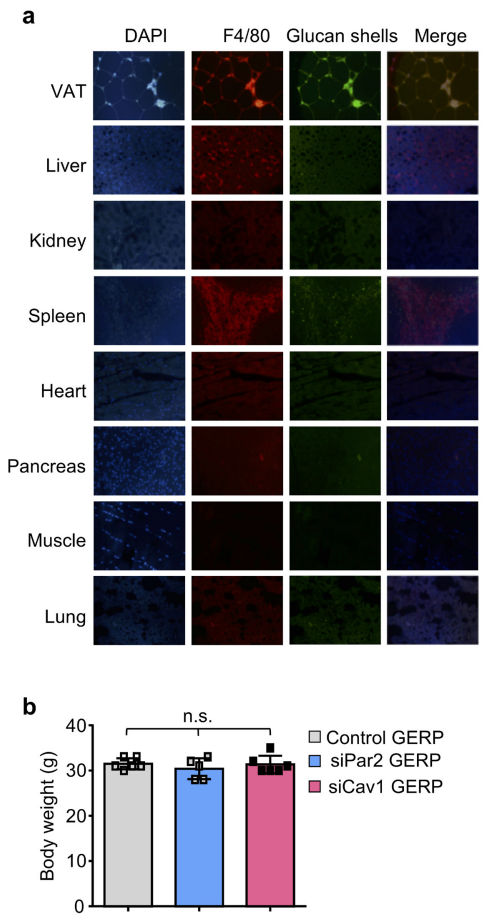
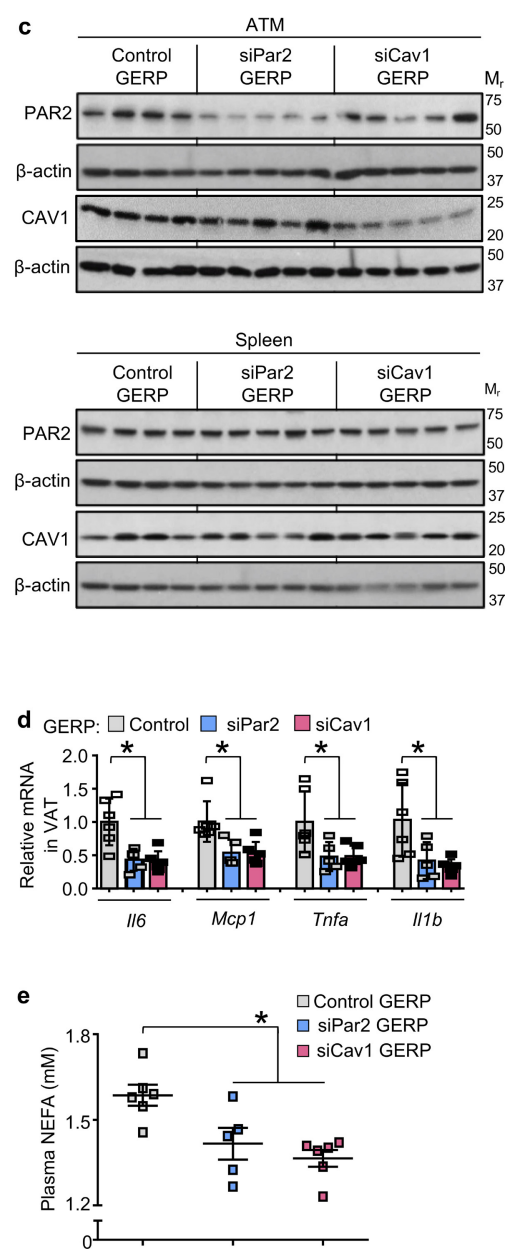

The mice were analysed $24 \mathrm{~h}$ after the last injection. b. Body weight. c, Immunoblots of PAR2 and CAV1 in ATMs and splenic extracts. d, Il6, $M c p 1$, Tnfa and $I l 1 b$ mRNA in VAT. e, Plasma non-esterified fatty acids (NEFA). In $\mathbf{b}, \mathbf{d}$ and $\mathbf{e}, n=5-6$ mice per group; $* P<0.05$ and n.s., nonsignificant by two-tailed Student's $t$-test for each siRNA GERP versus control GERP. For gel source data, see Supplementary Fig. 1. 


\section{natureresearch}

Corresponding author(s): Ira Tabas

\section{Life Sciences Reporting Summary}

Nature Research wishes to improve the reproducibility of the work that we publish. This form is intended for publication with all accepted life science papers and provides structure for consistency and transparency in reporting. Every life science submission will use this form; some list items might not apply to an individual manuscript, but all fields must be completed for clarity.

For further information on the points included in this form, see Reporting Life Sciences Research. For further information on Nature Research policies, including our data availability policy, see Authors \& Referees and the Editorial Policy Checklist.

Please do not complete any field with "not applicable" or n/a. Refer to the help text for what text to use if an item is not relevant to your study. For final submission: please carefully check your responses for accuracy; you will not be able to make changes later.

\section{- Experimental design}

\section{Sample size}

Describe how sample size was determined.

\section{Data exclusions}

Describe any data exclusions.

\section{Replication}

Describe the measures taken to verify the reproducibility of the experimental findings.

\section{Randomization}

Describe how samples/organisms/participants were allocated into experimental groups.

\section{Blinding}

Describe whether the investigators were blinded to group allocation during data collection and/or analysis.
Previous studies and pilot experiments form the basis of power calculations for the various studies. Depending on the experiment, calculations indicated that 3-12 mice per group would enable the testing of our hypotheses based on an expected $25-30 \%$ coefficient of variation and an $80 \%$ chance of detecting a 33\% difference in the key specified endpoints ( $P=0.05$ )

Pre-specified exclusion criteria were weight loss $>10 \%$ of initial body weight or signs of illness or injury requiring euthanasia. According to these pre-specified criteria, the maximum number of mice removed before analysis was 3 , but more typically $0-2$.

All experiments were reproducible as assessed in multiple wells of cells, tissue samples or mice. We did not have cases of irreproducibility. Please note that the bar graphs depicting spectral counts (Ext. Data Fig. 2 g and Ext. Data Fig. 8a) simply represent a single MS/MS experiment, and likewise the FPLC fractions (Ext. Data Fig. $2 f$ and Ext. Data Fig. 7c) were screened using technical duplicates, but all of these data were subjected to extensive reproducibility in all the subsequent biological assays related to these data.

Mice of the same age and similar weight were randomly assigned to experimental and control groups. On occasion, we analyzed a subset of mice for a particular parameter, and the subset was chosen randomly from a full cohort.

The investigators were not blinded during cell and mouse experiment assays. 
6. Statistical parameters

For all figures and tables that use statistical methods, confirm that the following items are present in relevant figure legends (or in the Methods section if additional space is needed).

$\mathrm{n} / \mathrm{a} \mid$ Confirmed

$\bigotimes$ The exact sample size $(n)$ for each experimental group/condition, given as a discrete number and unit of measurement (animals, litters, cultures, etc.)

$\checkmark$ A description of how samples were collected, noting whether measurements were taken from distinct samples or whether the same sample was measured repeatedly

$\bigotimes$ A statement indicating how many times each experiment was replicated

The statistical test(s) used and whether they are one- or two-sided

Only common tests should be described solely by name; describe more complex techniques in the Methods section.

$\searrow$ A description of any assumptions or corrections, such as an adjustment for multiple comparisons

Test values indicating whether an effect is present

Provide confidence intervals or give results of significance tests (e.g. P values) as exact values whenever appropriate and with effect sizes noted.

$\bigotimes$ A clear description of statistics including central tendency (e.g. median, mean) and variation (e.g. standard deviation, interquartile range) Clearly defined error bars in all relevant figure captions (with explicit mention of central tendency and variation)

See the web collection on statistics for biologists for further resources and guidance.

\section{- Software}

Policy information about availability of computer code

\section{Software}

Describe the software used to analyze the data in this study.

For manuscripts utilizing custom algorithms or software that are central to the paper but not yet described in the published literature, software must be made available to editors and reviewers upon request. We strongly encourage code deposition in a community repository (e.g. GitHub). Nature Methods guidance for providing algorithms and software for publication provides further information on this topic.

\section{- Materials and reagents}

Policy information about availability of materials

8. Materials availability

Indicate whether there are restrictions on availability of unique materials or if these materials are only available for distribution by a third party.

\section{Antibodies}

Describe the antibodies used and how they were validated for use in the system under study (i.e. assay and species).

10. Eukaryotic cell lines
a. State the source of each eukaryotic cell line used.
b. Describe the method of cell line authentication used.
c. Report whether the cell lines were tested for mycoplasma contamination.
d. If any of the cell lines used are listed in the database of commonly misidentified cell lines maintained by ICLAC, provide a scientific rationale for their use.

No restrictions--all mutant mice are commercially available or available from the labs indicated in the manuscript, and the methods for GeRP construction is published.

The source and catalogue number of all antibodies appear in Methods. Antibodies were validated by showing lack of signal after knockout or siRNA or by a single major band at the proper MW on immunoblot.

\section{- Animals and human research participants}

Policy information about studies involving animals; when reporting animal research, follow the ARRIVE guidelines

\section{Description of research animals}

Provide all relevant details on animals and/or

All details appear under "Mouse experiments" section in methods animal-derived materials used in the study. 
Policy information about studies involving human research participants

12. Description of human research participants

Describe the covariate-relevant population

N/A

characteristics of the human research participants. 